\title{
Investigation on the Self-synchronization of Dual Steady States for a Vibrating System with Four Unbalanced Rotors
}

Zhao Chunyu ( $\square$ chyzhao@mail.neu.edu.cn )

Northeastern University https://orcid.org/0000-0001-7237-6320

Mengchao Jiang

Northeastern University

Chunyu Zhao

Northeastern University

Yuanhao Wang

Northeastern University

Weihai Duan

Northeastern University

\section{Research Article}

Keywords: self-synchronization, coupling dynamics characteristics, ultra-resonance, sub-resonance, dual steady states

Posted Date: November 9th, 2021

DOI: https://doi.org/10.21203/rs.3.rs-1026423/v1

License: (1) This work is licensed under a Creative Commons Attribution 4.0 International License. Read Full License 


\section{Investigation on the self-synchronization of dual steady states for a vibrating system with four unbalanced rotors}

Mengchao Jiang, Chunyu Zhao*, Yuanhao Wang, Weihai Duan

School of Mechanical Engineering and Automation, Northeastern University, Shenyang 110819, China

Correspondence should be addressed to Chunyu Zhao; chyzhao@mail.neu.edu.cn

Abstract: In the field of vibration utilization engineering, to achieve the maximum degree or the highest efficiency use of the excitation force is still a hotspot among researchers. Based on this, this paper has carried out a series of synchronous theoretical analysis on the four identical unbalanced rotors (IURs) symmetrically and circularly mounted on a rigid frame (RF) model, which is used to drive a cone crusher. The dimensionless coupling equations of the four IURs are established using the improved small parameter method. The analysis of the coupling dynamics characteristics of the system shows that the four motors of the system adjust the speed through the synchronous torque to achieve synchronization, and a parameter determination method for realizing offset self-synchronization to eccentric force was put forward under the steady state of ultra-resonance. Furthermore, the relationship between the equivalent stiffness of the crushed material and crushing force and compression coefficient is discussed, and the design method of the full-load crusher working under the steady state of sub-resonance is proposed. Finally, through a series of computer simulations, the correctness of the self-synchronization of dual steady states is verified.

Keywords: self-synchronization; coupling dynamics characteristics; ultra-resonance; sub-resonance; dual steady states

\section{Introduction}

Self-synchronization of unbalanced rotors (URs), discovered at the Mekhanobr Institute in the Soviet Union in 1948, is a well-known non-linear phenomenon, whereby the synchronous regime 
arises due to natural properties of the processes themselves and their natural interaction [1]. Using the Poincare-Lyapunov method of small parameters and separation of direction motions, Blekhman gave physical explanation and mathematical description of self-synchronization phenomenon for URs [2-4] and developed the general theory to solve some problems of self-synchronization of URs in mechanical systems, such as self-synchronization of planetary URs on flatly-oscillating solid body, self-synchronization of the URs on the spatially-oscillating softly vibrato-isolated solid body, self-synchronization of the URs in systems with collisions of the bodies of the carrying vibrating system $[1,5]$. The two identical unbalanced rotors (IURs) vibrating systems' analytical approach can be greatly simplified by combining the differential equations of the two rotors with the differential equation of the phase difference between the two IURs, and its applications are to solve the self-synchronous problems for two IURs in circular motion, linear motion, centroid rotation motion and spatial motion vibrating machines [6-8]. The fresh opportunities have been opened up in the vibration technology by accomplishing these works, which have fascinated many researchers to develop vibrating mechanisms with specific dynamical parameters for improving the performances and motion modes of vibrating machines.

A simplest vibrating machine (VM) consists of a rigid frame (RF) on the elastic foundation, and two URs driven by two asynchronous motors, respectively. According to the ratio of the rotational frequency of the URs to the natural one of the VM, the operational states of VMs are classified into sub-resonance for that the ratio is much less than 1, near-resonance for that the ratio ranges from 0.95 to 1.05 , or ultra-resonance for that the ration is far greater than 1 . In an ultra-resonance vibrating system with two or multiple URs, the operating steady-state of system is determined by the principle of motion selection $[9,10]$. For a VM with two IURs symmetrically installed about the mass center of the RF, when the IURs rotate in the same direction, the motion of the RF is elliptical 
one for the case that ratio of the distance between the IURs and the mass center to the equivalent rotating radius of the $\mathrm{RF}$ is greater than $\sqrt{2}$, and swinging motion about its mass center for that less than $\sqrt{2}$ [11]. But when the two IURs rotate in opposite directions, the motion of the RF is linear one [12]. When the rotational axe of the two IURs are interspace lines and symmetrical about the symmetry axis of the RF, the trajectory of point on the RF is screw line $[7,13]$. When the rotational axe of the two URs are not symmetrical about the symmetry axis of the RF, the motion of the RF is translational one and swing of plane, and the trajectories of points on the RF are differential ellipses [14]. When two identical auxiliary rigid frames (ARFs) are symmetrically installed on a RF and two identical URs in the same direction are installed symmetrically on each of ARFs, the four IURs can excite the elliptical motion of the RF [10]. But when four IURs are installed on the same RF, exciting forces of the URs can be cancelled mutually and the RF is static under the condition of self-synchronization $[15,16]$. When three URs are in linear distribution on a RF, the three URs can achieve the RF's swinging and elliptical motion or linear motion by changing three URs' different installation methods [17-20], but the operation efficiency of vibrating system cannot be improved in the ultra-resonant state $[19,20]$. Besides, the anti-resonance system with three URs and two RFs is proposed to explore its motion selection [17, 18, 21]. It shows that the main rigid frame (MRF) can achieve swinging and elliptical motion while the accessorial rigid frame (ARF) is static by installing two URs on the same axis and symmetrically placing another UR on the other sides [17].

The models of double or multiple URs' self-synchronization have been applied increasingly to field of engineering machinery. Besides vibrating screens, vibrating feeders and vibrating conveyors [7-9, 22], a VM with two IURs located on two ARFs is proposed to achieve rock crushing in a crusher [23]. Shokhin et al discussed the effect of system parameters on crushing performances in [24]. 
In this paper, a single RF vibrating system with four IURs is proposed and applied to a cone crusher. This system operates two steady states of synchronization. One of synchronous states is ultra-resonance for idle state of the crusher, in which the exciting forces of the four IURs are cancelled mutually and the RF is static; another is sub-resonance for material crushing state, in which the exciting forces are supermposed mutually and excite the circular motion of the RF. In the next section, coupling dynamics analysis is done to derive dimensionless coupling equations of the four URs and obtain criteria of synchronization and stability for the four URs. The dynamic parameters of the VM that satisfy the two synchronous states are determined in section 4, and effect of crushed material property on the effective crushing force is discussed in section 5. In Section 6, computer simulation and experiments are conducted to verify the correctness of the theoretical research. Finally, conclusions are given in Section 7.

\section{Motion equations of system}

Figure 1 shows mechanism of a material crusher, which consists of a moving cone, a fixed cone, four supportors, and four driving systems. By means of a mandral, the fixed cone is supported on a rigid base on an elastic foundation. One driving system is composed of an asynchronous motor, a belt, a cardan shaft, a supporting shaft and a UR. One supportor is a connecting rod with two spherical linages at its ends. The moving cone is connected with the base by means of four supportors and driven by four symmetrically distributed IURs rotating with four asynchronous motors, respectively. When the system is running, the superimposed exciting forces stemming from the four URs cause the moving cone a circular motion on the horizontal plane, which periodically compresses the $\mathrm{V}$-shaped space between the moving cone and the fixed cone to achieves the purpose of crushing materials.

The overall structure of the vibration system is shown in Fig. 2 (a). At the radius $L$ of the moving 


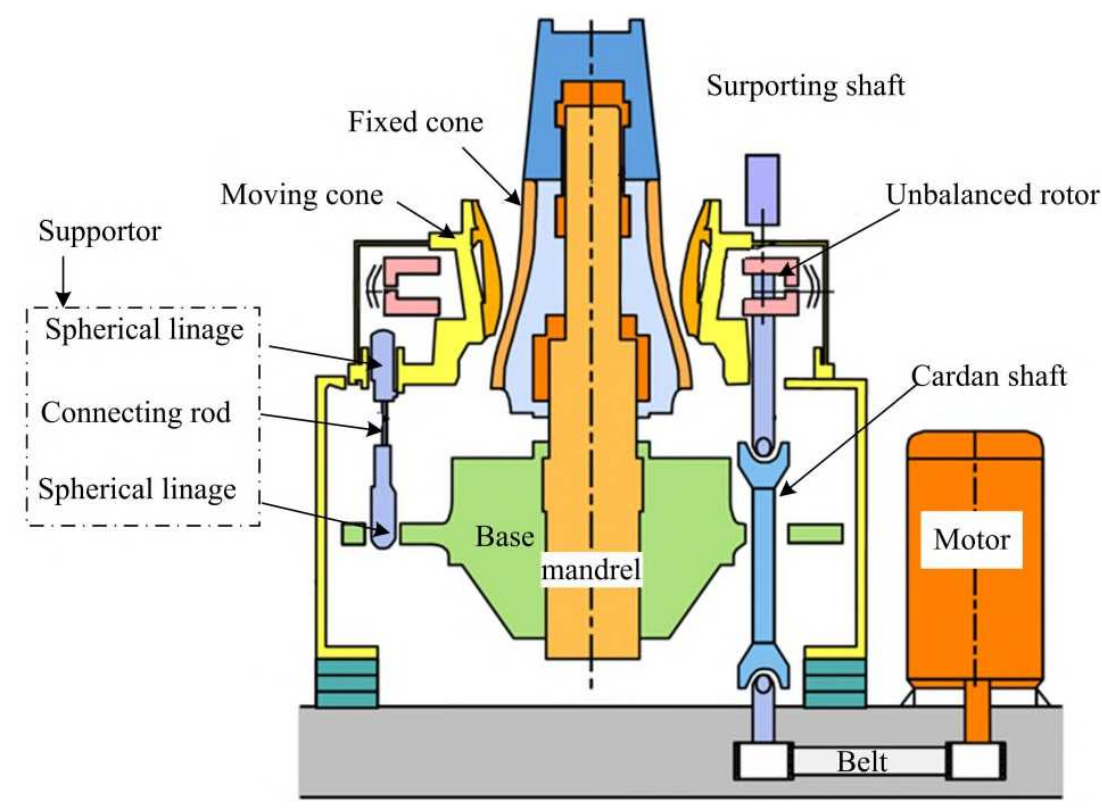

Fig. 1 Cone crusher and its 3D model

cone, we have to take the supportors' stiffness and damping into account. Their stiffness in the direction $x$ and $y$ the direction is respectively $k_{r x}, k_{r y}$, and their damping constant in the direction $x$ and $y$ is respectively $f_{r x}, f_{r y}$. The supportors dynamics model can be equivalent to shear rubber springs are installed as is illustrated in Fig. 2 (b), whose stiffness is $k_{r x} / 2$ in one direction of the $x$ axis and $k_{r y} / 2$ in one direction of the $y$ axis and the damping constant $f_{r x}$, $f_{r y}$.

To facilitate the calculation of the formula, we numbered the four IURs 1-4, among which the face to face mounting UR 1 and UR 2 are divided as a group, so do 3 and 4, as is shown in Fig. 2 (b). The system has three degrees of freedom, namely the movements in the $x$ and $y$ directions and the swing motion of $\psi$ around the center of mass $G$. The four IURs respectively rotate around their own motor shafts, and the angle of rotation is represented by $\varphi_{i}, i=1,2,3,4$.

In the coordinate system as is shown in Fig. 2 (c): oxy is the fixed coordinate system of the crusher; $G x^{\prime} y^{\prime}$ is the translational coordinate system and its $x^{\prime}$-axis and $y^{\prime}$-axis is parallel to the $x$-axis and $y$-axis respectively; $G x^{\prime \prime} y^{\prime \prime}$ is the follow-up coordinate system. The coordinates of the eccentric rotor of each UR (hereinafter referred to as an exciter) can be expressed as in $G x^{\prime \prime} y^{\prime \prime}$ : 


$$
\boldsymbol{x}_{i}^{\prime \prime}=\left\{\begin{array}{l}
R \cos \beta_{i}+r \cos \varphi_{i} \\
R \sin \beta_{i}+r \sin \varphi_{i}
\end{array}\right\}, \quad i=1,2,3,4
$$

where $R$ is the installation radius of the exciter center; $r$ is the eccentricity of the exciter with $r_{11}=r_{12}=r_{21}=r_{22}=r_{31}=r_{32}=r_{41}=r_{42}=r ; \beta_{i}$ is the angle between the center of the motor shaft and the $x$ axis with $\beta_{1}=0, \beta_{2}=\pi, \beta_{3}=\frac{\pi}{2}, \beta_{4}=\frac{3}{2} \pi$.

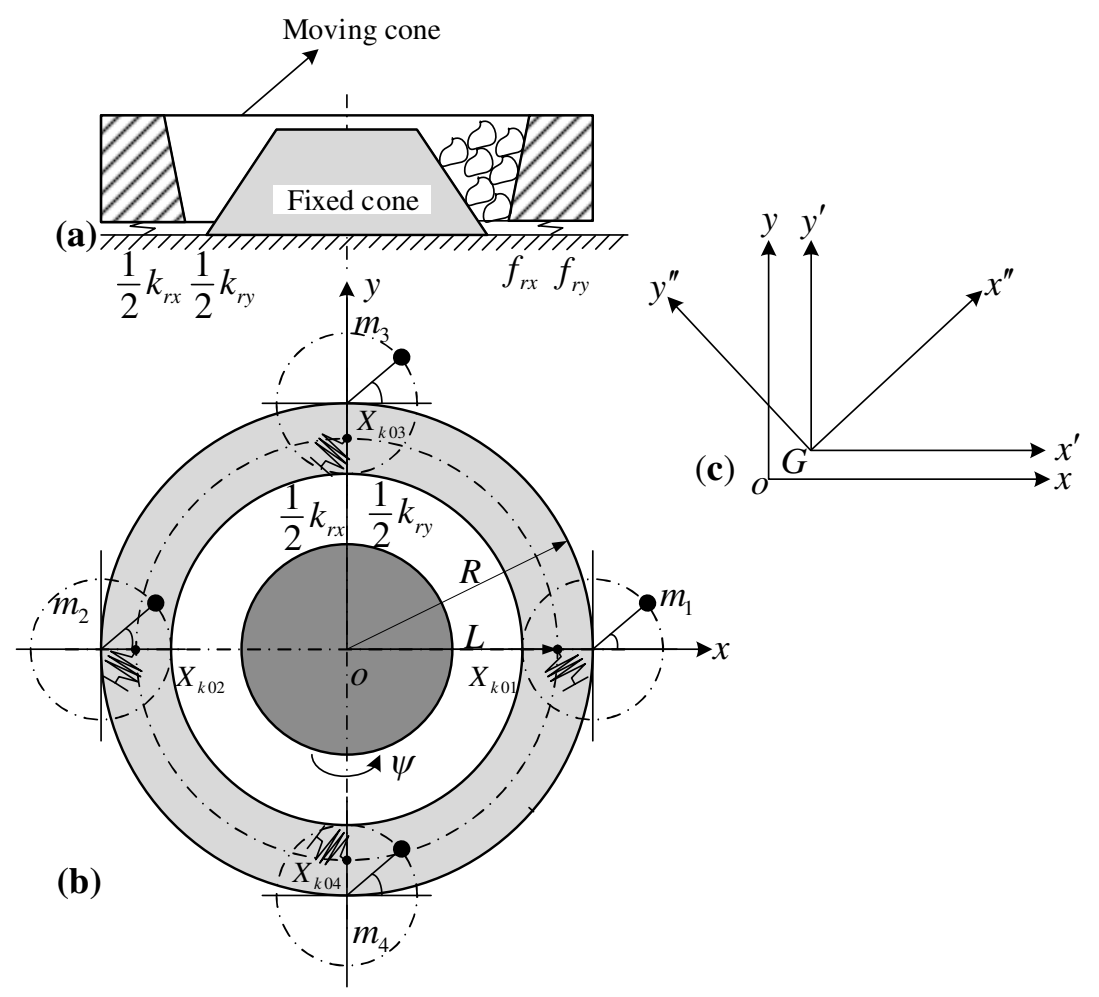

Fig. 2 (a) Vibration system structure; (b) The crusher's dynamic model; (c) Coordinate system

Using coordinate transformation, the coordinate of the exciters in the system $G x^{\prime \prime} y^{\prime \prime}$ is transformed into the system $G x^{\prime} y^{\prime}$ :

$$
\boldsymbol{x}_{i}^{\prime}=\boldsymbol{T} \boldsymbol{x}_{i}^{\prime \prime}, \quad i=1,2,3,4 \text {. }
$$

with the transformation matrix $\boldsymbol{T}=\left[\begin{array}{cc}\cos \psi & -\sin \psi \\ \sin \psi & \cos \psi\end{array}\right]$.

The coordinates in the fixed system oxy can be expressed as:

$$
\boldsymbol{x}_{i}=\boldsymbol{x}_{0}+\boldsymbol{T} \boldsymbol{x}_{i}^{\prime \prime}, \quad i=1,2,3,4 \text {. }
$$

with $\boldsymbol{x}_{0}=\{x, y\}^{T}$.

The coordinates of the joints between the springs and the RF are: 


$$
\boldsymbol{X}_{k i}=\boldsymbol{x}_{0}+\boldsymbol{T} \boldsymbol{X}_{k 0 i}, \quad i=1,2,3,4
$$

with $\boldsymbol{X}_{k 01}=\{-L, 0\}^{T}, \quad \boldsymbol{X}_{k 02}=\{L, 0\}^{T}, \quad \boldsymbol{X}_{k 03}=\{0, L\}^{T}, \quad \boldsymbol{X}_{k 04}=\{0,-L\}^{T}$.

According to the above coordinates and coordinate transformation, the kinetic energy and the potential energy of the system are respectively:

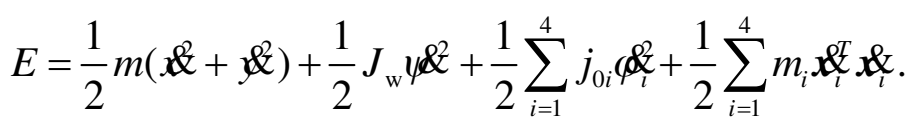

$$
\begin{aligned}
& V=\frac{1}{2} \sum_{i=1}^{4}\left(\boldsymbol{X}_{k i}-\boldsymbol{X}_{k 0 i}\right)^{T} \boldsymbol{K}_{i}\left(\boldsymbol{X}_{k i}-\boldsymbol{X}_{k 0 i}\right) \text {. }
\end{aligned}
$$

where $m$ is the mass of outer cone; $J_{\mathrm{w}}$ is the moment of inertia of outer cone; $j_{0 i}$ is the moment of inertia of motor $i ; m_{i}$ is the mass of each eccentric rotor with $m_{i}=m_{0}, i=1,2,3,4$; $\boldsymbol{K}_{i}$ is the stiffness matrix of rubber spring $i$ with $\boldsymbol{K}_{1}=\boldsymbol{K}_{2}=\operatorname{diag}\left(k_{r x} / 2,0\right), \quad \boldsymbol{K}_{3}=\boldsymbol{K}_{4}=\operatorname{diag}$ $\left(0, k_{r y} / 2\right)$

The energy dissipation of the system can be expressed as a function:

$$
D=\frac{1}{2} \sum_{i=1}^{4} \boldsymbol{X}_{k i}^{\ll T} \boldsymbol{F}_{i} \boldsymbol{\bigotimes}_{k i}^{\bigotimes}
$$

where $\boldsymbol{F}_{i}$ is the damping matrix of spring $i, \quad \boldsymbol{F}_{1}=\boldsymbol{F}_{2}=\operatorname{diag}\left(f_{r x} / 2,0\right), \quad \boldsymbol{F}_{3}=\boldsymbol{F}_{4}=\operatorname{diag}\left(0, f_{r y} / 2\right)$.

According to Lagrangian equation:

$$
\frac{\mathrm{d}}{\mathrm{d} t} \frac{\partial(E-V)}{\partial \phi_{l}}-\frac{\partial(E-V)}{\partial q_{i}}+\frac{\partial D}{\partial \&_{l}}=Q_{i} .
$$

In the vibrating system, we choose $q=\left\{x, y, \psi, \varphi_{1}, \varphi_{2}, \varphi_{3}, \varphi_{4}\right\}^{T}$ as the generalized coordinate of the system, under each generalized coordinate the generalized forces of the system can be expressed as $Q_{x}=Q_{y}=Q_{\psi}=0, Q_{\varphi i}=T_{e i}, i=1,2,3,4$. Substitute equations (5)-(7) into equation (8), in the calculation process, considering that the mass of each exciter of the exciter is much smaller than the mass of the vibrating rigid body, and the inertial coupling caused by the asymmetry of the installation of the exciter is ignored. And according to the literature $[6,7]$ reviewed, the rotation of the vibrating rigid body belongs to a small amplitude rotation, and the rotation angle satisfies 
$\psi<<1$, the differential equations of motion of the system are as follows:

$$
\begin{aligned}
& M f_{r x} \&+k_{r x} x=\sum_{i=1}^{4} m_{0} r\left(\phi_{i}^{2} \cos \varphi_{i}+\varphi_{l}^{\&} \sin \varphi_{i}\right), \\
& M f_{r y} \&+k_{r y} y=\sum_{i=1}^{4} m_{0} r\left(\phi_{i}^{\&} \sin \varphi_{i}-\operatorname{los}_{i} \cos \varphi_{i}\right) \text {, } \\
& J_{\psi} f_{\psi} \psi \&+k_{\psi} \psi=m_{0} R r \sum_{i=1}^{4}\left[\phi_{i}^{\&} \sin \left(\varphi_{i}-\beta_{i}\right)-\varphi_{i} \cos \left(\varphi_{i}-\beta_{i}\right)\right] \text {, } \\
& \left(j_{01}+m_{0} r^{2}\right)+f_{1} \&=T_{e 1}-m_{0} r\left\{\cos \varphi_{1}-\sin \varphi_{1}+\left[R \cos \left(\varphi_{1}-\beta_{1}\right)+r\right]\right. \\
& \left(j_{02}+m_{0} r^{2}\right)+f_{2} \&=T_{e 2}-m_{0} r\left\{\cos \varphi_{2}-\sin \varphi_{2}+\left[R \cos \left(\varphi_{2}-\beta_{2}\right)+r\right]\right. \\
& \left(j_{03}+m_{0} r^{2}\right)+f_{3} \&=T_{e 3}-m_{0} r\left\{\cos \varphi_{3}-\sin \varphi_{3}+\left[R \cos \left(\varphi_{3}-\beta_{3}\right)+r\right]\right. \\
& \left(j_{04}+m_{0} r^{2}\right)+f_{4} \&=T_{e 4}-m_{0} r\left\{\cos \varphi_{4}-\sin \varphi_{4}+\left[R \cos \left(\varphi_{4}-\beta_{4}\right)+r\right]\right.
\end{aligned}
$$

where $M=m+4 m_{0}, J_{\psi}=J_{\mathrm{w}}+4 m_{0}\left(R^{2}+r^{2}\right)=M l_{\mathrm{e}}^{2}, f_{\psi}=\frac{1}{2}\left(f_{r x}+f_{r y}\right) L^{2}, k_{\psi}=\frac{1}{2}\left(k_{r x}+k_{r y}\right) L^{2} ; f_{i}$ is the shaft damping constant of motor $i ; T_{\mathrm{e} i}$ is the output electromagnetic torque of motor $i ; l_{\mathrm{e}}$ is the equivalent radius of gyration of the system; $(4)$ are the derivatives with respect to time $t$.

\section{Synchronization of the four IURs system}

Assume that the average phase between the four exciters is $\varphi$, the phase difference between the exciter 1 and the exciter 2 is $2 \alpha_{1}$, the phase difference between the exciter 3 and the exciter 4 is $2 \alpha_{2}$, the phase difference between the exciters $1 \& 2$ and the exciters $3 \& 4$ is $2 \alpha_{3}$, which satisfies:

$$
\varphi=\frac{1}{4} \sum_{i=1}^{4} \varphi_{i}, 2 \alpha_{1}=\varphi_{1}-\varphi_{2}, 2 \alpha_{2}=\varphi_{3}-\varphi_{4}, 2 \alpha_{3}=\frac{1}{2}\left(\sum_{m=1}^{2} \varphi_{m}-\sum_{n=3}^{4} \varphi_{n}\right)
$$

Assuming the average value of the average angular velocity of the four exciters is $\omega_{\mathrm{m}}$, the instantaneous fluctuation coefficient of the average angular velocity $\& \&$ is $\varsigma_{0}$, the instantaneous fluctuation coefficient of $a_{i}^{\&}$ is $\varsigma_{i}$, then the small parameters are set as:

$$
\begin{gathered}
\phi \&=\left(1+\varsigma_{0}\right) \omega_{\mathrm{m}}, \\
\alpha_{l}^{\&}=\varsigma_{i} \omega_{\mathrm{m}}, i=1,2,3 .
\end{gathered}
$$

Combining the above formula, the phase, instantaneous angular velocity and instantaneous angular acceleration of the four exciters can be expressed as: 


$$
\begin{gathered}
\varphi_{1}=\varphi+\alpha_{1}+\alpha_{3}=\varphi+\vartheta_{1}, \\
\varphi_{2}=\varphi-\alpha_{1}+\alpha_{3}=\varphi+\vartheta_{2}, \\
\varphi_{3}=\varphi+\alpha_{2}-\alpha_{3}=\varphi+\vartheta_{3}, \\
\varphi_{4}=\varphi-\alpha_{2}-\alpha_{3}=\varphi+\vartheta_{4}, \\
\alpha \&=\left(1+\varsigma_{0}+\varsigma_{1}+\varsigma_{3}\right) \omega_{\mathrm{m}}=\left(1+\varepsilon_{1}\right) \omega_{\mathrm{m}}, \\
\& \&=\left(1+\varsigma_{0}-\varsigma_{1}+\varsigma_{3}\right) \omega_{\mathrm{m}}=\left(1+\varepsilon_{2}\right) \omega_{\mathrm{m}}, \\
\& \&=\left(1+\varsigma_{0}-\varsigma_{1}+\varsigma_{3}\right) \omega_{\mathrm{m}}=\left(1+\varepsilon_{2}\right) \omega_{\mathrm{m}}, \\
\& \&=\left(1+\varsigma_{0}+\varsigma_{2}-\varsigma_{3}\right) \omega_{\mathrm{m}}=\left(1+\varepsilon_{3}\right) \omega_{\mathrm{m}}, \\
\& \&=\left(1+\varsigma_{0}-\varsigma_{2}-\varsigma_{3}\right) \omega_{\mathrm{m}}=\left(1+\varepsilon_{4}\right) \omega_{\mathrm{m}}, \\
\& \&=\& \omega_{\mathrm{m}}, \quad \& \omega_{\mathrm{m}}, \quad \& \omega_{\mathrm{m}}, \quad \& \omega_{\mathrm{m}} .
\end{gathered}
$$

where $\varepsilon_{i}, i=1,2,3,4$ is the instantaneous fluctuation coefficient of the angular velocity of each exciter for respectively. If the average value $\bar{\varepsilon}_{i}$ in a single positive period satisfies $\bar{\varepsilon}_{i}=0$, then the running speeds of the four exciters are the same, that is, the operation is synchronized. When the vibration system is in a stable state of synchronous operation, the term in the differential equations of motion in the $x, y$, and $\psi$ directions, that is, the effect of angular velocity on the steady state excitation can be ignored $[6,7]$. Therefore, the equation in the steady state of the system can be written as:

$$
\begin{gathered}
2 \xi_{x} \omega_{\mathrm{n} x} \&+\omega_{\mathrm{n} x}^{2} x=r_{\mathrm{m}} r \omega_{\mathrm{m}}^{2} \sum_{i=1}^{4}\left(1+\varepsilon_{i}\right)^{2} \cos \left(\varphi+\vartheta_{i}\right), \\
2 \xi_{y} \omega_{\mathrm{n} y} \&+\omega_{\mathrm{n} y}^{2} y=r_{\mathrm{m}} r \omega_{\mathrm{m}}^{2} \sum_{i=1}^{4}\left(1+\varepsilon_{i}\right)^{2} \sin \left(\varphi+\vartheta_{i}\right), \\
\frac{2 \xi_{\psi} \omega_{\mathrm{n} \psi}}{l_{\mathrm{e}}^{2}} \psi \&+\frac{\omega_{\mathrm{n} \psi}^{2}}{l_{\mathrm{e}}^{2}} \psi=\frac{r_{\mathrm{m}} r_{\mathrm{e}} r}{l_{\mathrm{e}}} \omega_{\mathrm{m}}^{2} \sum_{i=1}^{4}\left(1+\varepsilon_{i}\right)^{2} \sin \left(\varphi+\vartheta_{i}-\beta_{i}\right) .
\end{gathered}
$$

where

$$
\begin{gathered}
r_{\mathrm{m}}=\frac{m_{0}}{M}, l_{\mathrm{e}}=\sqrt{\frac{J_{\psi}}{M}}, r_{\mathrm{e}}=\frac{R}{l_{\mathrm{e}}}, \omega_{\mathrm{n} x}=\sqrt{\frac{k_{r x}}{M}}, \omega_{\mathrm{n} y}=\sqrt{\frac{k_{r y}}{M}}, \omega_{\mathrm{n} \psi}=\sqrt{\frac{k_{\psi}}{M}}, \quad \xi_{x}=\frac{f_{r x}}{2 \sqrt{k_{r x} M}}, \\
\xi_{y}=\frac{f_{r y}}{2 \sqrt{k_{r y} M}}, \quad \xi_{\psi}=\frac{f_{\psi}}{2 \sqrt{k_{\psi} M}} .
\end{gathered}
$$

Considering the damping coefficient of the spring, The fluctuation coefficient of the speed of each motor $\varepsilon_{i}$ is too small to affect the excitation response in all directions. Therefore, according to the superposition principle of the linear system, the response in each direction can be approximately 
expressed as the following form:

$$
\begin{gathered}
x=r_{\mathrm{m}} r \mu_{x} \sum_{i=1}^{4} \cos \left(\varphi+\vartheta_{i}-\gamma_{x}\right), \\
y=r_{\mathrm{m}} r \mu_{y} \sum_{i=1}^{4} \sin \left(\varphi+\vartheta_{i}-\gamma_{y}\right), \\
\psi=\frac{r_{\mathrm{m}} r}{r_{\mathrm{e}} r \mu_{\psi}} \sum_{i=1}^{4} \sin \left(\varphi+\vartheta_{i}-\beta_{i}-\gamma_{\psi}\right) .
\end{gathered}
$$

where $\mu_{x}=\frac{n_{x}^{2}}{\sqrt{\left(1-n_{x}^{2}\right)^{2}+\left(2 \xi_{x} n_{x}\right)^{2}}}, \quad \mu_{y}=\frac{n_{y}^{2}}{\sqrt{\left(1-n_{y}^{2}\right)^{2}+\left(2 \xi_{y} n_{y}\right)^{2}}}, \quad \mu_{\psi}=\frac{n_{\psi}^{2}}{\sqrt{\left(1-n_{\psi}^{2}\right)^{2}+\left(2 \xi_{\psi} n_{\psi}\right)^{2}}}$ means the vibration amplification factor of the system. In addition, $n_{x}, n_{y}, n_{\psi}$ represents the ratio of the vibration frequency to the natural frequency in each direction, we have:

$$
n_{x}=\frac{\omega_{\mathrm{m}}}{\omega_{\mathrm{n} x}}, n_{y}=\frac{\omega_{\mathrm{m}}}{\omega_{\mathrm{n} y}}, n_{\psi}=\frac{\omega_{\mathrm{m}}}{\omega_{\mathrm{n} \psi}}
$$

And $\gamma_{x}, \gamma_{y}, \gamma_{\psi}$ represents the phase lag angles in three directions, satisfying $\gamma=\operatorname{tg}^{-1} \frac{2 \xi n}{1-n^{2}}$.

From the formula (16), we can get the expression of $\&$, $\psi \&$, Noticing $\quad \&+\mathscr{Q}_{i}=\left(1+\varepsilon_{i}\right) \omega_{\mathrm{m}}$,

Substitute them into the equation of the unbalanced rotor of the differential equation of motion (9),

Integrate $\varphi$ on $0-2 \pi$ and divide by $2 \pi$ to get the average value in a single period, after ignoring the higher-order terms of $\varepsilon_{i}$ and \&, we obtain:

$$
\begin{aligned}
& \left(j_{01}+m_{0} r^{2}\right) \stackrel{\mathrm{E}}{\mathrm{E}} \omega_{\mathrm{m}}+f_{1}\left(1+\bar{\varepsilon}_{1}\right) \omega_{\mathrm{m}} \\
& =\bar{T}_{\mathrm{e} 1}-m_{0} r^{2} \omega_{\mathrm{m}}\left(\sum_{j=1}^{4}\left(\chi_{1 j}^{\prime} \mathrm{\varepsilon}_{j}^{\mathrm{e}}+\chi_{1 j} \bar{\varepsilon}_{j}\right)+\chi_{\mathrm{f} 1}+\chi_{\mathrm{a} 1}\right), \\
& \left(j_{02}+m_{0} r^{2}\right) \stackrel{\mathbb{E}}{\&} \omega_{\mathrm{m}}+f_{2}\left(1+\bar{\varepsilon}_{2}\right) \omega_{\mathrm{m}} \\
& =\bar{T}_{\mathrm{e} 2}-m_{0} r^{2} \omega_{\mathrm{m}}\left(\sum_{j=1}^{4}\left(\chi_{2 j}^{\prime} \mathrm{\epsilon}_{j}^{\mathrm{E}}+\chi_{2 j} \bar{\varepsilon}_{j}\right)+\chi_{\mathrm{f} 2}+\chi_{\mathrm{a} 2}\right) \text {, } \\
& \left(j_{03}+m_{0} r^{2}\right) \stackrel{\&}{\&} \omega_{\mathrm{m}}+f_{3}\left(1+\bar{\varepsilon}_{3}\right) \omega_{\mathrm{m}} \\
& =\bar{T}_{\mathrm{e} 3}-m_{0} r^{2} \omega_{\mathrm{m}}\left(\sum_{j=1}^{4}\left(\chi_{3 j}^{\prime} \mathrm{\varepsilon}_{j}^{\mathrm{E}}+\chi_{3 j} \bar{\varepsilon}_{j}\right)+\chi_{\mathrm{f} 3}+\chi_{\mathrm{a} 3}\right) \text {, } \\
& \left(j_{04}+m_{0} r^{2}\right) \stackrel{\mathrm{E}}{\mathrm{E}} \omega_{\mathrm{m}}+f_{4}\left(1+\bar{\varepsilon}_{4}\right) \omega_{\mathrm{m}} \\
& =\bar{T}_{\mathrm{e} 4}-m_{0} r^{2} \omega_{\mathrm{m}}\left(\sum_{j=1}^{4}\left(\chi_{4 j}^{\prime} \mathrm{E}_{j}^{\mathrm{E}}+\chi_{4 j} \bar{\varepsilon}_{j}\right)+\chi_{\mathrm{f} 4}+\chi_{\mathrm{a} 4}\right) \text {. }
\end{aligned}
$$

The $\chi_{i j}^{\prime}, \chi_{i j}, \chi_{\mathrm{f} i}, \chi_{\mathrm{a} i}$ in equation (16) are given in the Appendix A. It is worth noting that in 
equation (16), compared with the changes of the angle of rotation, the changes of $\alpha_{1}, \alpha_{2}, \alpha_{3}$ to time are small, so $\varepsilon_{i}(i=1,2,3,4)$ can be regarded as a slowly varying parameter. Therefore, in the above integration process, according to the method of direct separation of motion [1-5], we replace $\varepsilon_{i}, \underset{i}{\&}, \alpha_{j}, j=1,2,3$ with its average value $\bar{\varepsilon}_{i}, \varepsilon_{i}^{\mathrm{d}}, \bar{\alpha}_{j}, j=1,2,3$. In the equation (16), when the rotation speed of the induction motor is in a steady-state operating state near, the electromagnetic torque of each motor can be expressed as:

$$
\begin{aligned}
& \bar{T}_{\mathrm{e} 1}=T_{\mathrm{e} 01}-\bar{k}_{\mathrm{e} 01} \bar{\varepsilon}_{1}, \\
& \bar{T}_{\mathrm{e} 2}=T_{\mathrm{e} 02}-\bar{k}_{\mathrm{e} 02} \bar{\varepsilon}_{2}, \\
& \bar{T}_{\mathrm{e} 3}=T_{\mathrm{e} 03}-\bar{k}_{\mathrm{e} 03} \bar{\varepsilon}_{3}, \\
& \bar{T}_{\mathrm{e} 4}=T_{\mathrm{e} 04}-\bar{k}_{\mathrm{e} 04} \bar{\varepsilon}_{4} .
\end{aligned}
$$

When the four motors are powered by the same source and have the same number of pole pairs, in the stator voltage synchronous coordinate system, they can be expressed as:

$$
\begin{gathered}
T_{\mathrm{e} 0 i}=n_{\mathrm{p}} \frac{L_{\mathrm{m} i}^{2} U_{\mathrm{s} 0}^{2}}{L_{\mathrm{s} i}^{2} R_{\mathrm{r} i}} \frac{\omega_{\mathrm{s}}-n_{\mathrm{p}} \omega_{\mathrm{m}}}{1+\sigma_{i}^{2} \tau_{\mathrm{r} i}^{2}\left(\omega_{\mathrm{s}}-n_{\mathrm{p}} \omega_{\mathrm{m}}\right)^{2}}, i=1,2,3,4 . \\
k_{\mathrm{e} 0 i}=n_{\mathrm{p}}^{2} \frac{L_{\mathrm{m} i}^{2} U_{\mathrm{s} 0}^{2}}{L_{\mathrm{s} i}^{2} \omega_{\mathrm{s}} R_{\mathrm{r} i}} \frac{1-\sigma_{i}^{2} \tau_{\mathrm{r} i}^{2}\left(\omega_{\mathrm{s}}-n_{\mathrm{p}} \omega_{\mathrm{m}}\right)^{2}}{\left[1+\sigma_{i}^{2} \tau_{\mathrm{r} i}^{2}\left(\omega_{\mathrm{s}}-n_{\mathrm{p}} \omega_{\mathrm{m}}\right)^{2}\right]^{2}} \frac{\omega_{\mathrm{m}}}{\omega_{\mathrm{s}}}, i=1,2,3,4 .
\end{gathered}
$$

where $R_{\mathrm{s} i}, R_{\mathrm{r} i}$ represents the stator resistance and the rotor resistance; $L_{\mathrm{s} i}, L_{\mathrm{r} i}, L_{\mathrm{m} i}$ represents the stator inductance, the rotor inductance and the mutual inductance; $n_{\mathrm{p}}$ represents the number of pole pairs of the motor; $\tau_{\mathrm{r} i}$ represents the rotor time constant with $\tau_{\mathrm{r} i}=L_{\mathrm{r} i} / R_{\mathrm{r} i} ; \sigma_{i}$ represents the asynchronous motor leakage inductance constant with $\sigma_{i}=1-L_{\mathrm{m} i}^{2} / L_{\mathrm{s} i} L_{\mathrm{r} i} ; \omega_{\mathrm{s}}$ represents the grid power supply frequency; $U_{\mathrm{s} 0}$ represents the terminal voltage.

In the equation (16), since the moments of inertia of the motor shaft $j_{0 i}, i=1,2,3,4$ are much smaller than $m_{0} r^{2}$, they are ignored in the calculation. Substituting the equation (17) into (16) can obtain the differential equations for the small parameters $\bar{\varepsilon}_{1}, \overline{\varepsilon_{2}}, \overline{\varepsilon_{3}}, \bar{\varepsilon}_{4}$.Using $m_{0} r^{2} \omega_{\mathrm{m}}$ to divide both sides of the equation and introduce the following dimensionless parameters:

$$
\rho_{0}=1+W_{\mathrm{c} 0} / 2
$$




$$
\begin{aligned}
& \kappa_{11}=\frac{\bar{k}_{\mathrm{e} 01}}{m_{0} r^{2} \omega_{\mathrm{m}}^{2}}+\frac{f_{1}}{m_{0} r^{2} \omega_{\mathrm{m}}}+W_{\mathrm{s} 0}, \\
& \kappa_{22}=\frac{\bar{k}_{\mathrm{e} 02}}{m_{0} r^{2} \omega_{\mathrm{m}}^{2}}+\frac{f_{2}}{m_{0} r^{2} \omega_{\mathrm{m}}}+W_{\mathrm{s} 0}, \\
& \kappa_{33}=\frac{\bar{k}_{\mathrm{e} 03}}{m_{0} r^{2} \omega_{\mathrm{m}}^{2}}+\frac{f_{3}}{m_{0} r^{2} \omega_{\mathrm{m}}}+W_{\mathrm{s} 0}, \\
& \kappa_{44}=\frac{\bar{k}_{\mathrm{e} 04}}{m_{0} r^{2} \omega_{\mathrm{m}}^{2}}+\frac{f_{4}}{m_{0} r^{2} \omega_{\mathrm{m}}}+W_{\mathrm{s} 0} .
\end{aligned}
$$

From this, the matrix form of the dimensionless coupling equation can be obtained

$$
\boldsymbol{A} \mathcal{E}=\boldsymbol{B} \bar{\varepsilon}+\boldsymbol{u} .
$$

with $\overline{\boldsymbol{\varepsilon}}=\left\{\bar{\varepsilon}_{1}, \bar{\varepsilon}_{2}, \bar{\varepsilon}_{3}, \bar{\varepsilon}_{4}\right\}^{T}$ and $\boldsymbol{u}=\left\{u_{1}, u_{2}, u_{3}, u_{4}\right\}^{T}$;

$$
\begin{aligned}
\boldsymbol{A}=\left[\begin{array}{cccc}
\rho_{0} & \chi_{12}^{\prime} & \chi_{13}^{\prime} & \chi_{14}^{\prime} \\
\chi_{21}^{\prime} & \rho_{0} & \chi_{23}^{\prime} & \chi_{24}^{\prime} \\
\chi_{31}^{\prime} & \chi_{32}^{\prime} & \rho_{0} & \chi_{34}^{\prime} \\
\chi_{41}^{\prime} & \chi_{42}^{\prime} & \chi_{43}^{\prime} & \rho_{0}
\end{array}\right], \boldsymbol{B}=-\omega_{\mathrm{m}}\left[\begin{array}{cccc}
\kappa_{11} & \chi_{12} / \omega_{\mathrm{m}} & \chi_{13} / \omega_{\mathrm{m}} & \chi_{14} / \omega_{\mathrm{m}} \\
\chi_{21} / \omega_{\mathrm{m}} & \kappa_{22} & \chi_{23} / \omega_{\mathrm{m}} & \chi_{24} / \omega_{\mathrm{m}} \\
\chi_{31} / \omega_{\mathrm{m}} & \chi_{32} / \omega_{\mathrm{m}} & \kappa_{33} & \chi_{34} / \omega_{\mathrm{m}} \\
\chi_{41} / \omega_{\mathrm{m}} & \chi_{42} / \omega_{\mathrm{m}} & \chi_{43} / \omega_{\mathrm{m}} & \kappa_{44}
\end{array}\right], \\
u_{1}=\frac{T_{\mathrm{e} 01}}{m_{0} r^{2} \omega_{\mathrm{m}}}-\frac{f_{1}}{m_{0} r^{2}}-\chi_{\mathrm{f} 1}-\chi_{\mathrm{a} 1}, \\
u_{2}=\frac{T_{\mathrm{e} 02}}{m_{0} r^{2} \omega_{\mathrm{m}}}-\frac{f_{2}}{m_{0} r^{2}}-\chi_{\mathrm{f} 2}-\chi_{\mathrm{a} 2}, \\
u_{3}=\frac{T_{\mathrm{e} 03}}{m_{0} r^{2} \omega_{\mathrm{m}}}-\frac{f_{3}}{m_{0} r^{2}}-\chi_{\mathrm{f} 3}-\chi_{\mathrm{a} 3}, \\
u_{4}=\frac{T_{\mathrm{e} 04}}{m_{0} r^{2} \omega_{\mathrm{m}}}-\frac{f_{4}}{m_{0} r^{2}}-\chi_{\mathrm{f} 4}-\chi_{\mathrm{a} 4} .
\end{aligned}
$$

where $\boldsymbol{A}$ is the dimensionless inertia coupling matrix, $\boldsymbol{B}$ is the angular velocity dimensionless stiffness coupling matrix, $\boldsymbol{u}$ is the dimensionless load coupling.

\subsection{Synchronization conditions}

If the four vibration exciters of the vibration system of the vibrating crusher realize synchronous operation, then $\bar{\varepsilon}_{i}=0, i=1,2,3,4$. In the matrix form of the dimensionless coupling equation, we have $\overline{\boldsymbol{\varepsilon}}=\boldsymbol{0}, \quad \mathcal{\&}=\boldsymbol{0}$, the equation (20) can be simplified to $\boldsymbol{u}=\boldsymbol{0}$, and let:

$$
\begin{aligned}
& T_{\mathrm{o} 1}=T_{\mathrm{e} 01}-f_{1} \omega_{\mathrm{m}}=m_{0} r^{2} \omega_{\mathrm{m}}\left(\chi_{\mathrm{f} 1}+\chi_{\mathrm{a} 1}\right), \\
& T_{\mathrm{o} 2}=T_{\mathrm{e} 02}-f_{2} \omega_{\mathrm{m}}=m_{0} r^{2} \omega_{\mathrm{m}}\left(\chi_{\mathrm{f} 2}+\chi_{\mathrm{a} 2}\right), \\
& T_{\mathrm{o} 3}=T_{\mathrm{e} 03}-f_{3} \omega_{\mathrm{m}}=m_{0} r^{2} \omega_{\mathrm{m}}\left(\chi_{\mathrm{f} 3}+\chi_{\mathrm{a} 3}\right), \\
& T_{\mathrm{o} 4}=T_{\mathrm{e} 04}-f_{4} \omega_{\mathrm{m}}=m_{0} r^{2} \omega_{\mathrm{m}}\left(\chi_{\mathrm{f} 4}+\chi_{\mathrm{a} 4}\right) .
\end{aligned}
$$


$T_{\mathrm{o} 1}-T_{\mathrm{o} 4}$ represent the output electromagnetic torques of four motors during synchronization, the vibration system transfers electromagnetic torque between the four exciters by adjusting the phase difference between the four exciters to balance the difference in output torque between the four motors. Introduce the output torque difference:

$$
\begin{aligned}
\Delta T_{\mathrm{o} 12}= & T_{\mathrm{o} 1}-T_{\mathrm{o} 2} \\
= & \left(m_{0} r^{2} \omega_{\mathrm{m}}^{2} / 2\right)\left[W_{\mathrm{sc} 13} \cos \left(\bar{\alpha}_{1}-\bar{\alpha}_{2}+2 \bar{\alpha}_{3}\right)+W_{\mathrm{sc} 14} \cos \left(\bar{\alpha}_{1}+\bar{\alpha}_{2}+2 \bar{\alpha}_{3}\right)+2 W_{\mathrm{cc}} \sin 2 \bar{\alpha}_{1}\right. \\
& -W_{\mathrm{sc} 23} \cos \left(2 \bar{\alpha}_{3}-\bar{\alpha}_{1}-\bar{\alpha}_{2}\right)-W_{\mathrm{sc} 24} \cos \left(2 \bar{\alpha}_{3}-\bar{\alpha}_{1}+\bar{\alpha}_{2}\right)+W_{\mathrm{cc} 13} \sin \left(\bar{\alpha}_{1}-\bar{\alpha}_{2}+2 \bar{\alpha}_{3}\right) \\
& \left.+W_{\mathrm{cc} 14} \sin \left(\bar{\alpha}_{1}+\bar{\alpha}_{2}+2 \bar{\alpha}_{3}\right)-W_{\mathrm{cc} 23} \sin \left(2 \bar{\alpha}_{3}-\bar{\alpha}_{1}-\bar{\alpha}_{2}\right)-W_{\mathrm{cc} 24} \sin \left(2 \bar{\alpha}_{3}-\bar{\alpha}_{1}+\bar{\alpha}_{2}\right)\right], \\
\Delta T_{\mathrm{o} 34}= & T_{\mathrm{o} 3}-T_{\mathrm{o} 4} \\
= & \left(m_{0} r^{2} \omega_{\mathrm{m}}^{2} / 2\right)\left[W_{\mathrm{sc} 13} \cos \left(\bar{\alpha}_{1}-\bar{\alpha}_{2}+2 \bar{\alpha}_{3}\right)+W_{\mathrm{sc} 23} \cos \left(2 \bar{\alpha}_{3}-\bar{\alpha}_{1}-\bar{\alpha}_{2}\right)+2 W_{\mathrm{cc}} \sin 2 \bar{\alpha}_{2}\right. \\
& -W_{\mathrm{sc} 14} \cos \left(\bar{\alpha}_{1}+\bar{\alpha}_{2}+2 \bar{\alpha}_{3}\right)-W_{\mathrm{sc} 24} \cos \left(2 \bar{\alpha}_{3}-\bar{\alpha}_{1}+\bar{\alpha}_{2}\right)-W_{\mathrm{cc} 13} \sin \left(\bar{\alpha}_{1}-\bar{\alpha}_{2}+2 \bar{\alpha}_{3}\right) \\
& \left.-W_{\mathrm{cc} 23} \sin \left(2 \bar{\alpha}_{3}-\bar{\alpha}_{1}-\bar{\alpha}_{2}\right)+W_{\mathrm{cc} 14} \sin \left(\bar{\alpha}_{1}+\bar{\alpha}_{2}+2 \bar{\alpha}_{3}\right)+W_{\mathrm{cc} 24} \sin \left(2 \bar{\alpha}_{3}-\bar{\alpha}_{1}+\bar{\alpha}_{2}\right)\right], \\
\Delta T_{\mathrm{o}}= & T_{\mathrm{o} 1}+T_{\mathrm{o} 22}-T_{\mathrm{o} 3}-T_{o 4} \\
= & m_{0} r^{2} \omega_{\mathrm{m}}^{2}\left[W_{\mathrm{sc}} \cos 2 \bar{\alpha}_{1}-W_{\mathrm{sc}} \cos 2 \bar{\alpha}_{2}+W_{\mathrm{cc} 13} \sin \left(\bar{\alpha}_{1}-\bar{\alpha}_{2}+2 \bar{\alpha}_{3}\right)\right. \\
& \left.+W_{\mathrm{cc} 23} \sin \left(2 \bar{\alpha}_{3}-\bar{\alpha}_{1}-\bar{\alpha}_{2}\right)+W_{\mathrm{cc} 14} \sin \left(\bar{\alpha}_{1}+\bar{\alpha}_{2}+2 \bar{\alpha}_{3}\right)+W_{\mathrm{cc} 24} \sin \left(2 \bar{\alpha}_{3}-\bar{\alpha}_{1}+\bar{\alpha}_{2}\right)\right] .
\end{aligned}
$$

Let $T_{\mathrm{su}}=\frac{m_{0} r^{2} \omega_{\mathrm{m}}^{2}}{2}$ denotes the kinetic energy of the exciter with an eccentric rotor. According to equations (25)-(27), we have:

$$
\begin{aligned}
& \frac{\Delta T_{\mathrm{o} 12}}{T_{\mathrm{su}}}=\tau_{\mathrm{c} 12}\left(\bar{\alpha}_{1}, \bar{\alpha}_{2}, \bar{\alpha}_{3}\right), \\
& \frac{\Delta T_{\mathrm{o} 34}}{T_{\mathrm{su}}}=\tau_{\mathrm{c} 34}\left(\bar{\alpha}_{1}, \bar{\alpha}_{2}, \bar{\alpha}_{3}\right), \\
& \frac{\Delta T_{\mathrm{o}}}{T_{\mathrm{su}}}=\tau_{\mathrm{c}}\left(\bar{\alpha}_{1}, \bar{\alpha}_{2}, \bar{\alpha}_{3}\right) .
\end{aligned}
$$

where $\tau_{\mathrm{c} 12}\left(\bar{\alpha}_{1}, \bar{\alpha}_{2}, \bar{\alpha}_{3}\right), \tau_{\mathrm{c} 34}\left(\bar{\alpha}_{1}, \bar{\alpha}_{2}, \bar{\alpha}_{3}\right), \tau_{\mathrm{c}}\left(\bar{\alpha}_{1}, \bar{\alpha}_{2}, \bar{\alpha}_{3}\right)$ shows the dimensionless coupling torque between exciters 1 and 2, exciters 3 and 4, and exciters 12 and 34 respectively. The left side of equations (29)-(31) represents the difference of the dimensionless residual electromagnetic torques. The dimensionless coupling torques are function of $\bar{\alpha}_{1}, \bar{\alpha}_{2}, \bar{\alpha}_{3}$, which satisfies:

$$
\begin{aligned}
& \left|\tau_{\mathrm{c} 12}\left(\bar{\alpha}_{1}, \bar{\alpha}_{2}, \bar{\alpha}_{3}\right)\right| \leq \tau_{\mathrm{c} 12 \max }, \\
& \left|\tau_{\mathrm{c} 34}\left(\bar{\alpha}_{1}, \bar{\alpha}_{2}, \bar{\alpha}_{3}\right)\right| \leq \tau_{\mathrm{c} 34 \max },
\end{aligned}
$$




$$
\left|\tau_{\mathrm{c}}\left(\bar{\alpha}_{1}, \bar{\alpha}_{2}, \bar{\alpha}_{3}\right)\right| \leq \tau_{\mathrm{cmax}}
$$

Among them, $\tau_{\mathrm{c} 12 \max }, \tau_{\mathrm{c} 34 \max }, \tau_{\mathrm{cmax}}$ respectively represents the maximum value of three groups of non-dimensional coupling moments. In order to ensure the synchronous operation of the four exciters, it is necessary to ensure that the above equation has a solution. Therefore, the synchronization criterion for the synchronous operation of the four exciters is transformed into the existence of the solution of the phase difference equation between the exciters. The synchronization criterions are as follows:

$$
\left|\frac{\Delta T_{\mathrm{o} 12}}{T_{\mathrm{su}}}\right| \leq \tau_{\mathrm{c} 12 \max },\left|\frac{\Delta T_{\mathrm{o} 34}}{T_{\mathrm{su}}}\right| \leq \tau_{\mathrm{c} 34 \max },\left|\frac{\Delta T_{\mathrm{o}}}{2 T_{\mathrm{su}}}\right| \leq \tau_{\mathrm{cmax}} .
$$

Define the maximum value of the dimensionless coupling torques as the synchronous torques or the capture torques between the groups of exciters, the meaning of the synchronization criterions is: the synchronous torques between the exciters are not less than the absolute value of the difference between the corresponding dimensionless residual electromagnetic torques.

\subsection{Stability of synchronization and stable criteria}

When $\boldsymbol{u}=\boldsymbol{0}$, the above system is the generalized system [25]:

$$
A_{0} \&=B_{0} \varepsilon
$$

Where $\boldsymbol{A}_{0}$ and $\boldsymbol{B}_{0}$ are the values of $\boldsymbol{A}$ and $\boldsymbol{B}$ for $\bar{\alpha}_{1}=\bar{\alpha}_{10}, \bar{\alpha}_{2}=\bar{\alpha}_{20}, \bar{\alpha}_{3}=\bar{\alpha}_{30}$ and $\omega_{\mathrm{m}}=\omega_{\mathrm{m} 0}$. When the system is under no-load, it is operating in the state of far beyond resonance, the phase lag angle in all directions is close to $\pi, W_{\mathrm{s} 0}, W_{\mathrm{sc}}$ can be ignored, $\boldsymbol{A}_{0}$ is symmetrical and $\boldsymbol{B}_{0}$ is anti-symmetric, when the parameters of the vibrating system satisfy the follow condition:

$$
\begin{gathered}
a_{i j}>0, \quad i=1,2,3,4 ; j=1,2,3,4 . \\
\operatorname{det}\left(\boldsymbol{A}_{02}\right)>0, \operatorname{det}\left(\boldsymbol{A}_{03}\right)>0, \\
\operatorname{det}\left(\boldsymbol{A}_{0}\right)>0 .
\end{gathered}
$$

the matrices $\boldsymbol{A}_{0}$ and $\boldsymbol{B}_{0}$ satisfy the generalized Lyapunov equations[25]: 


$$
\begin{gathered}
\boldsymbol{I}^{\mathrm{T}} \boldsymbol{B}_{0}+\boldsymbol{B}_{0}^{\mathrm{T}} \boldsymbol{I}=-\omega_{\mathrm{m} 0} \operatorname{diag}\left\{\kappa_{11}, \kappa_{22}, \kappa_{33}, \kappa_{44}\right\}, \\
\boldsymbol{A}_{0}^{\mathrm{T}} \boldsymbol{I}=\boldsymbol{I} \boldsymbol{A}_{0}>0 .
\end{gathered}
$$

where $\boldsymbol{I}$ is the unit matrix.

If satisfies $\lim \underset{t \rightarrow \infty}{A \boldsymbol{\varepsilon}}=\boldsymbol{0}$, then the above-mentioned generalized system is permissible and impulsive-free, and the generalized system is in a stable state, so when $\lim _{t \rightarrow \infty}^{\boldsymbol{\varepsilon}}=\boldsymbol{0}$, it means that the electromagnetic torque of the four motors and the load torque applied by the vibration system are balanced. Linearizing equations $(21)-(24)$ around $\bar{\alpha}_{10}, \bar{\alpha}_{20}, \bar{\alpha}_{30}, \omega_{\mathrm{m} 0}$, and neglecting $W_{\mathrm{s} 0}$, $W_{\mathrm{sc}}, f_{1}-f_{4}$, we obtain:

$$
\begin{gathered}
k_{\mathrm{e} 01}\left(\varsigma_{0}+\varsigma_{1}+\varsigma_{3}\right)=-\sum_{i=1}^{3}\left(\frac{\partial \chi_{\mathrm{a} 1}}{\partial \bar{\alpha}_{i}}\right)_{0} \Delta \alpha_{i}, \\
k_{\mathrm{e} 02}\left(\varsigma_{0}-\varsigma_{1}+\varsigma_{3}\right)=-\sum_{i=1}^{3}\left(\frac{\partial \chi_{\mathrm{a} 2}}{\partial \bar{\alpha}_{i}}\right)_{0} \Delta \alpha_{i}, \\
k_{\mathrm{e} 03}\left(\varsigma_{0}+\varsigma_{2}-\varsigma_{3}\right)=-\sum_{i=1}^{3}\left(\frac{\partial \chi_{\mathrm{a} 3}}{\partial \bar{\alpha}_{i}}\right)_{0} \Delta \alpha_{i}, \\
k_{\mathrm{e} 04}\left(\varsigma_{0}-\varsigma_{2}-\varsigma_{3}\right)=-\sum_{i=1}^{3}\left(\frac{\partial \chi_{\mathrm{a} 4}}{\partial \bar{\alpha}_{i}}\right)_{0} \Delta \alpha_{i} .
\end{gathered}
$$

where $(\bullet)_{0}$ are the values of $(\bullet)$ for $\bar{\alpha}_{1}=\bar{\alpha}_{10}, \bar{\alpha}_{2}=\bar{\alpha}_{20}, \bar{\alpha}_{3}=\bar{\alpha}_{30} ;$ and $\Delta \alpha_{i}=\bar{\alpha}_{i}-\bar{\alpha}_{i 0}$, $i=1,2,3,4$. Summing up equations (39)-(42), we obtain:

$$
\varsigma_{0}=\rho_{1} \varsigma_{1}+\rho_{2} \varsigma_{2}+\rho_{3} \varsigma_{3}
$$

where $\rho_{1}=\frac{k_{\mathrm{e} 02}-k_{\mathrm{e} 01}}{k_{\mathrm{e} 01}+k_{\mathrm{e} 02}+k_{\mathrm{e} 03}+k_{\mathrm{e} 04}}, \quad \rho_{2}=\frac{k_{\mathrm{e} 04}-k_{\mathrm{e} 03}}{k_{\mathrm{e} 01}+k_{\mathrm{e} 02}+k_{\mathrm{e} 03}+k_{\mathrm{e} 04}}, \quad \rho_{3}=\frac{k_{\mathrm{e} 03}+k_{\mathrm{e} 04}-k_{\mathrm{e} 01}-k_{\mathrm{e} 02}}{k_{\mathrm{e} 01}+k_{\mathrm{e} 02}+k_{\mathrm{e} 03}+k_{\mathrm{e} 04}}$.

Substituting equation (43) into equations (39) - (42), the left side of the equations has $\varsigma_{i}=\frac{\Delta a_{i}}{\omega_{\mathrm{m} 0}}$, $i=1,2,3$. By subtracting equation (40) from (39) as the first row, subtracting equation (42) from (41) as the second row, subtracting the sum of equations (41) and (42) from that of equations (39) and (40) as the third row, we can obtain the generalized system with respect to $\Delta \alpha=\left\{\Delta \alpha_{1}, \Delta \alpha_{2}, \Delta \alpha_{3}\right\}^{T}$ : 
where $\boldsymbol{E}=\left[e_{i j}\right]_{3 \times 3}, \quad \boldsymbol{D}=\left[d_{i j}\right]_{3 \times 3}$ are specifically given in Appendix B.

Generalized system (44) can be rewritten as:

$$
\Delta \alpha=C \Delta \alpha, C=E^{-1} D
$$

Solving $\operatorname{det}(\boldsymbol{C}-\lambda \boldsymbol{I})=0$, the characteristic equation of $\boldsymbol{C}$ can be obtained:

$$
\lambda^{3}+a_{1} \lambda^{2}+a_{2} \lambda+a_{3}=0
$$

Only when all characteristic roots have negative real parts, the zero-valued solutions of (45) are stable. According to the Routh-Hurwitz criterion, the coefficients of the above characteristic equation need to meet the following conditions:

$$
a_{1}>0, a_{3}>0, a_{1} a_{2}>a_{3} .
$$

In the system of this paper, the parameters of four induction motors are selected to be similar:

$$
\begin{gathered}
k_{\mathrm{e} 01} \approx k_{\mathrm{e} 02} \approx k_{\mathrm{e} 03} \approx k_{\mathrm{e} 04} \approx k_{\mathrm{e} 0}, \\
T_{\mathrm{e} 1} \approx T_{\mathrm{e} 2} \approx T_{\mathrm{e} 3} \approx T_{\mathrm{e} 4} .
\end{gathered}
$$

In this case, the matrix $\boldsymbol{E}$ can be simplified to:

$$
\boldsymbol{E}=\operatorname{diag}\left\{2 k_{\mathrm{e} 0}, 2 k_{\mathrm{e} 0}, 4 k_{\mathrm{e} 0}\right\}
$$

After multiplying the inverse matrix of $\boldsymbol{E}$ and the matrix $\boldsymbol{D}, \boldsymbol{C}$ it can be written as follows:

$$
\boldsymbol{C}=\left[c_{i j}\right]_{3 \times 3}=\left[\begin{array}{lll}
d_{11} / 2 k_{\mathrm{e} 0} & d_{12} / 2 k_{\mathrm{e} 0} & d_{13} / 2 k_{\mathrm{e} 0} \\
d_{21} / 2 k_{\mathrm{e} 0} & d_{22} / 2 k_{\mathrm{e} 0} & d_{23} / 2 k_{\mathrm{e} 0} \\
d_{31} / 4 k_{\mathrm{e} 0} & d_{32} / 4 k_{\mathrm{e} 0} & d_{33} / 4 k_{\mathrm{e} 0}
\end{array}\right] .
$$

The coefficients of the characteristic equation (46) satisfy the following relationship:

$$
\begin{gathered}
a_{1}=-c_{11}-c_{22}-c_{33}, \\
a_{2}=-c_{12}^{2}-\frac{1}{2} c_{23}^{2}-\frac{1}{2} c_{13}^{2}+c_{11} c_{22}+c_{22} c_{33}+c_{33} c_{11}, \\
a_{3}=-c_{11} c_{22} c_{33}-\frac{1}{2} c_{12} c_{23} c_{13}-\frac{1}{2} c_{13} c_{12} c_{23}+\frac{1}{2} c_{11} c_{23}^{2}+\frac{1}{2} c_{22} c_{13}^{2}+c_{33} c_{12}^{2} .
\end{gathered}
$$

The system must meet the Routh-Hurwitz criterion after satisfying the Lyapunov criterion.

$$
\left\{\begin{array}{l}
2 \alpha_{1}=2 \alpha_{2} \in(-\pi / 2, \pi / 2), W_{\mathrm{cc}}>0 \\
2 \alpha_{1}=2 \alpha_{2} \in(\pi / 2,3 \pi / 2), W_{\mathrm{cc}}<0
\end{array}\right.
$$

The subsequent numerical analysis results can be verified at this point. According to the Lyapunov 
stability criterion and the value of the cosine coupling coefficient $W_{\mathrm{cc}}$, the value range of steady-state phase differences $2 \bar{\alpha}_{1}, 2 \bar{\alpha}_{2}$ can be given as (52).

\section{Numerical analysis}

In order to prove the self-synchronization ability and stability of the vibrating system for idle state, it is necessary to analyze the system in the related synchronization problems and the stable state numerically. The parameters of the vibrating system selected in numerical analysis are: mass of outer cone $m=1260 \mathrm{~kg}$, mass of eccentric rotor $m_{0}=60 \mathrm{~kg}$, that is $r_{\mathrm{m}}=0.04, r=0.2 \mathrm{~m}, R=1.6$ $\mathrm{m}, L=1.2 \mathrm{~m}$. The frequency is under the ultra-resonance condition when the system is load-free, we take the frequency ratio of each direction as $n_{x}=n_{y}=n_{\psi}=4$. For vibrating machine, we take the rubber spring damping ratio as $\xi_{x}=\xi_{y}=\xi_{\psi}=0.07[6,7]$. The selected four motors are exactly the same, which are three-phase squirrel cage asynchronous motors $(380 \mathrm{~V}, 50 \mathrm{~Hz}, 6$-pole, $\Delta$ -connection, rated power $7.5 \mathrm{~kW}$, rated speed $980 \mathrm{r} / \mathrm{min}$ ), stator resistance $R_{\mathrm{s}}=3.35 \Omega$, rotor resistance $R_{\mathrm{r}}=3.40 \Omega$, stator inductance $L_{\mathrm{s}}=170 \mathrm{mH}$, rotor inductance $L_{\mathrm{r}}=170 \mathrm{mH}$, mutual inductance $L_{\mathrm{m}}=164 \mathrm{mH}, f_{1}=f_{2}=f_{3}=f_{4}=0.05$.

\subsection{Steady-state phase differences for idle state}

Substitute the above data into equations (21) - (24) and obtain nonlinear equations related to the parameters $r_{\mathrm{m}}, r_{\mathrm{e}}, \mu_{x}, \mu_{y}, \mu_{\psi}, \omega_{\mathrm{m} 0}, \bar{\alpha}_{10}, \bar{\alpha}_{20}, \bar{\alpha}_{30}$, where $r_{\mathrm{m}}, \mu_{x}, \mu_{y}, \mu_{\psi}$ has been given by system parameters. Solving them by numerical method, we can obtain the stable motor speed $\omega_{\mathrm{m} 0}$ and the phase differences $\bar{\alpha}_{10}, \bar{\alpha}_{20}, \bar{\alpha}_{30}$ when the system operates synchronization, the solutions of the phase difference are:

$$
\begin{gathered}
2 \bar{\alpha}_{10}=0, \pm \pi, \\
2 \bar{\alpha}_{20}=0, \pm \pi, \\
2 \bar{\alpha}_{30}=0, \pm \pi / 2, \pm \pi .
\end{gathered}
$$

In order to reflect the influence of the dimensionless parameter $r_{\mathrm{e}}$ on the synchronization and 
stability of the system more intuitively, it is necessary to discuss the maximum value of it:

$$
r_{\mathrm{e}}^{2}=\left(\frac{R}{l_{\mathrm{e}}}\right)^{2}=\frac{1}{\left(l_{\mathrm{ew}} / R\right)^{2}+4 r_{\mathrm{m}}\left(1+r^{2} / R^{2}\right)}, \quad r_{\mathrm{e} \text { ax }}^{2}=\lim _{R \rightarrow+\infty} r_{\mathrm{e}}^{2}=1 / 4 r_{\mathrm{m}}
$$

where $l_{\mathrm{ew}}=\sqrt{J_{\mathrm{w}} / M}$. So according to the given mass ratio $r_{\mathrm{m}}=0.04$, we can get the $r_{\mathrm{emax}}$ value of 2.5, that is, in the analysis of synchronization and stability, the value of $r_{\mathrm{e}}$ should be limited to between 0 and its maximum value of 2.5 .

Combining the Lyapunov stability criterion (36), the Routh-Hurwitz stability criterion (47) and the value range of $r_{\mathrm{e}}$ to determine the phase difference combination in (53), we can obtain the relationship between the dimensionless parameter $r_{\mathrm{e}}$ and the steady-state phase differences as shown in Fig. 3. The synchronous speed of the system under steady-state phase differences can be calculated through the solution process above, is at the stable value of $\omega_{\mathrm{m} 0}=104.2 \mathrm{rad} / \mathrm{s}$.

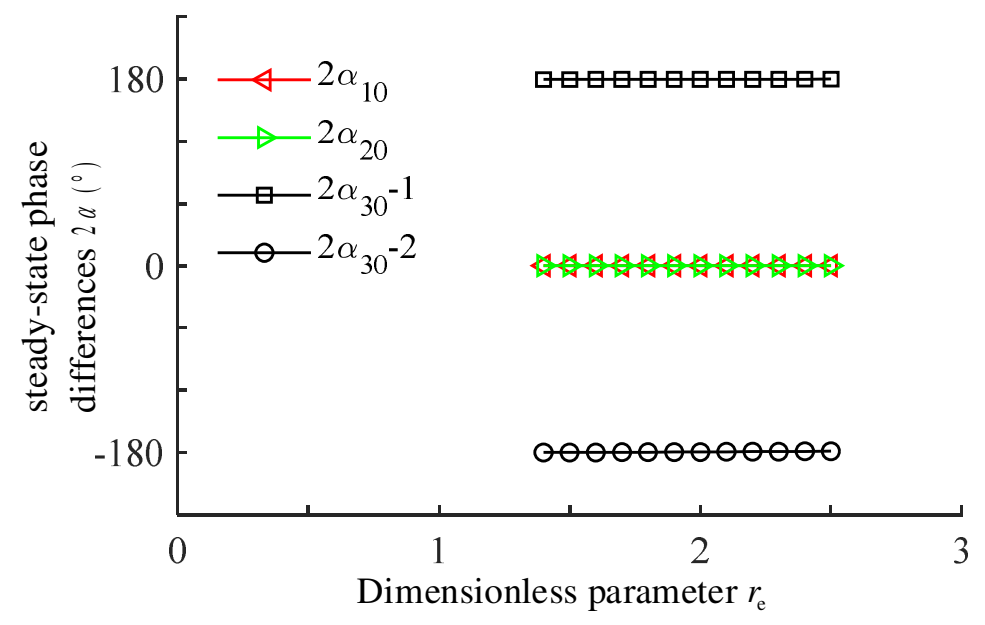

Fig. 3 Stable phase differences versus $r_{\mathrm{e}}$ for idle state

As shown in Fig. 3, when the ratio of the installation radius of the system exciter to the equivalent radius of gyration of the system $r_{\mathrm{e}}$ satisfies $1.414<r_{\mathrm{e}}<2.5$, the phase difference between the exciter 1 and the exciter $22 \bar{\alpha}_{10}$ and the phase difference between the exciter 3 and the exciter 4 $2 \bar{\alpha}_{20}$ are all around 0 . The phase difference between the exciters $1 \& 2$ and the exciters $3 \& 4$ has two steady-state values $2 \bar{\alpha}_{30}-1$ and $2 \bar{\alpha}_{30}-2$ in this parameter interval, respectively stable nearly $\pi$ and $-\pi$. In this case, according to the principle of linear superposition of the excitation response 
(15), the excitation responses of the four exciters cancel each other out. The excitation responses in the three directions are 0 so that the system does not move (When the three groups of phase differences deviate slightly from 0 or $\pm \pi$, it is a slight vibration). Explain it with the principle of the movement selection theory, $2 \bar{\alpha}_{10}=2 \bar{\alpha}_{20}=0$ makes $x, y, \psi$ responses superimpose and the system selects the circular motion and the rotation of the rigid body. While $2 \bar{\alpha}_{30}= \pm \pi$, the $x, y$ and $\psi$ directions of the forces applied by the two sets of exciters are opposite. Therefore, the steady-state phase differences combination as shown in Fig. 3 makes the rigid body in a non-moving state.

When $r_{\mathrm{e}}<1.414,2 \bar{\alpha}_{10}=2 \bar{\alpha}_{20}= \pm \pi$, the $x$ and $y$ directions are in non-moving state; $2 \bar{\alpha}_{30}=0$ or $\pm \pi$ makes the system select the movement in the $\psi$ direction, the energy consumption of the system is equal in the two states 0 or $\pm \pi$ of $2 \bar{\alpha}_{30}$, this kind of choice of $2 \bar{\alpha}_{30}$ is uncertain, so the system does not have stable phase differences under the $r_{\mathrm{e}}<1.414$ parameter setting. This verifies of the results of the stability judgment prediction in section 2 .

\subsection{Stability curve}

Applying the above numerical analysis to the characteristic equation of matrix $\boldsymbol{C}$, we obtain the three characteristic roots of $C$. Take the real part of the three characteristic roots as $\lambda_{1}, \lambda_{2}, \lambda_{3}$ and regard them as the first stability coefficients of the system. By plotting the first stability coefficients' curve, the general trend of stability with the change of $r_{\mathrm{e}}$ can be obtained. It can be seen from Fig. 4 that when the dimensional parameter $r_{\mathrm{e}}$ of the system change from minimum to maximum, the coefficients $\lambda_{1}, \lambda_{2}, \lambda_{3}$ are always less than $0 . \lambda_{1}$ maintains at around -2.45 , while $\lambda_{2}$ and $\lambda_{3}$ are equal and gradually decrease with the increase of $r_{\mathrm{e}}$. The first stability coefficients are based on the Routh-Hurwitz criterion, and the smaller the negative real part of the eigenvalue, the stronger the stability of the system. Considering along with the Lyapunov criterion, the elements $a_{i j}$ in matrix $\boldsymbol{A}^{\prime}$ and the leading principle minors: 


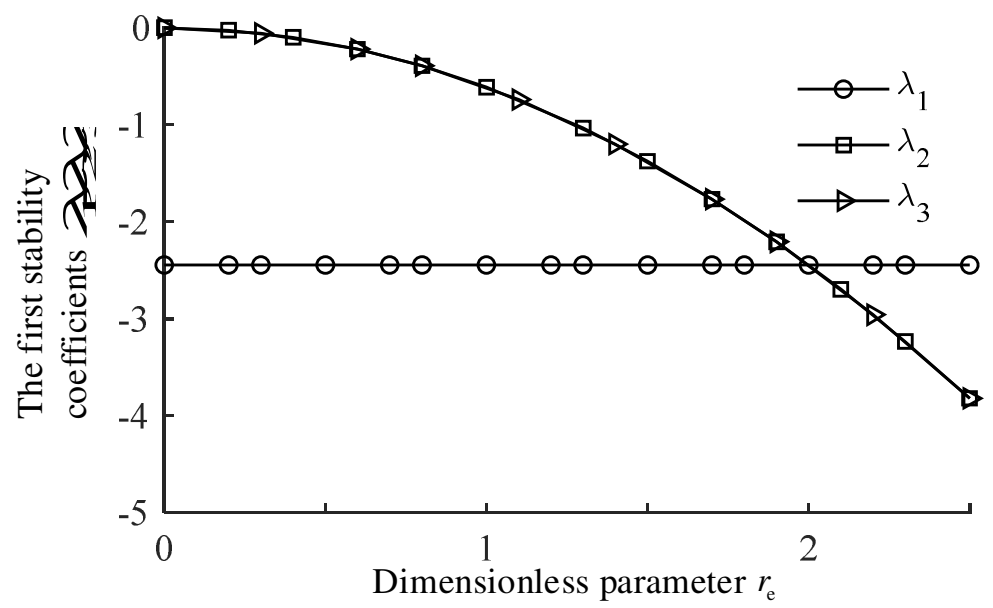

Fig. 4 The first stability coefficients for idle state of $r_{\mathrm{m}}=0.04$
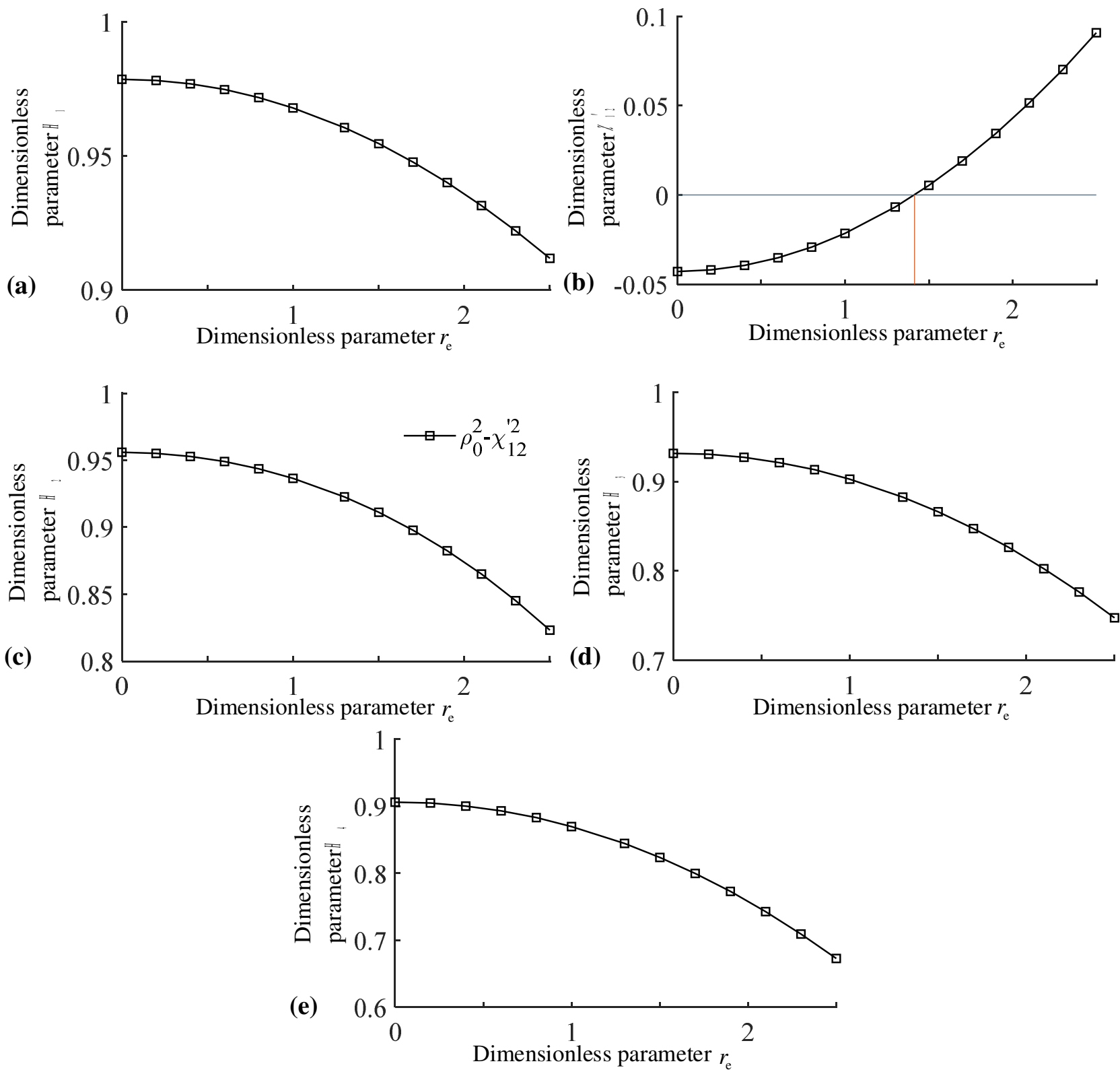

Fig. 5 The second stability coefficients for idle state of $r_{\mathrm{m}}=0.04$; (a) $H_{1}$; (b) $\chi_{12}^{\prime}$ (other elements in matrix $\boldsymbol{A}^{\prime}$ are constants greater than 0 );
(c) $\mathrm{H}_{2}$;
(d) $\mathrm{H}_{3}$;
(e) $H_{4}$.

$H_{1}=\rho_{0}$ of order 1, $H_{2}=\operatorname{det}\left(\boldsymbol{A}_{02}\right)$ of order $2, H_{3}=\operatorname{det}\left(\boldsymbol{A}_{03}\right)$ of order 3, $H_{4}=\operatorname{det}\left(\boldsymbol{A}_{04}\right)$ of order 
4 are regarded as the second stability coefficients. Fig. 5 shows all the second stability coefficients.

Fig. 5 (b) shows that the dimensionless parameter $\chi_{12}^{\prime}$ is greater than 0 when $r_{\mathrm{e}}$ is greater than 1.414, which verifies the accuracy of numerical calculation of the steady-state phase differences. From Fig. 5 (a) and Fig. 5 (c) -(e), it can be seen that all other dimensionless parameters except for $\chi_{12}^{\prime}$ are greater than 0 for all feasible values of $r_{\mathrm{e}} \cdot \chi_{12}^{\prime}$ are the decisive factors affecting the stability. Moreover, what affects $\chi_{12}^{\prime}$ is the cosine coupling coefficient $W_{\text {cc }}$, which should be mentioned. Selecting $r_{\mathrm{e}}$ to ensure $W_{\mathrm{cc}}>0$, the stability for idle state can be achieved.

\subsection{Synchronous capability curve}

The greater the coupling torques of each group of exciters, the greater the torque differences between the drive motors are allowed when the system implements synchronization. Introduce the synchronous ability coefficients to indicate the ability of the exciters to achieve synchronization. Substituting $\chi_{\mathrm{f} i}, \quad \chi_{\mathrm{a} i}$ and the kinetic energy of an unbalanced rotor $T_{\mathrm{su}}=m_{0} r^{2} \omega_{\mathrm{m}}^{2} / 2$ into equations $(21)-(24)$, and using $\bar{\vartheta}_{1}, \bar{\vartheta}_{2}, \bar{\vartheta}_{3}, \bar{\vartheta}_{4}$ to express the phase differences $\overline{\alpha_{1}}, \overline{\alpha_{2}}, \bar{\alpha}_{3}$, we obtain:

$$
\begin{aligned}
T_{01}= & T_{\mathrm{su}}\left[W_{\mathrm{s} 0}+W_{\mathrm{sc}} \cos \left(\bar{\vartheta}_{1}-\bar{\vartheta}_{2}\right)+W_{\mathrm{sc} 13} \cos \left(\bar{\vartheta}_{1}-\bar{\vartheta}_{3}\right)+W_{\mathrm{sc} 14} \cos \left(\bar{\vartheta}_{1}-\bar{\vartheta}_{4}\right)\right. \\
& \left.+W_{\mathrm{cc}} \sin \left(\bar{\vartheta}_{1}-\bar{\vartheta}_{2}\right)+W_{\mathrm{cc} 13} \sin \left(\bar{\vartheta}_{1}-\bar{\vartheta}_{3}\right)+W_{\mathrm{cc} 14} \sin \left(\bar{\vartheta}_{1}-\bar{\vartheta}_{4}\right)\right] \\
T_{02}= & T_{\mathrm{su}}\left[W_{\mathrm{s} 0}+W_{\mathrm{sc}} \cos \left(\bar{\vartheta}_{1}-\bar{\vartheta}_{2}\right)+W_{\mathrm{sc} 23} \cos \left(\bar{\vartheta}_{2}-\bar{\vartheta}_{3}\right)+W_{\mathrm{sc} 24} \cos \left(\bar{\vartheta}_{2}-\bar{\vartheta}_{4}\right)\right. \\
& \left.-W_{\mathrm{cc}} \sin \left(\bar{\vartheta}_{1}-\bar{\vartheta}_{2}\right)+W_{\mathrm{cc} 23} \sin \left(\bar{\vartheta}_{2}-\bar{\vartheta}_{3}\right)+W_{\mathrm{cc} 24} \sin \left(\bar{\vartheta}_{2}-\bar{\vartheta}_{4}\right)\right] \\
T_{03}= & T_{\mathrm{su}}\left[W_{\mathrm{s} 0}+W_{\mathrm{sc} 13} \cos \left(\bar{\vartheta}_{1}-\bar{\vartheta}_{3}\right)+W_{\mathrm{sc} 23} \cos \left(\bar{\vartheta}_{2}-\bar{\vartheta}_{3}\right)+W_{\mathrm{sc}} \cos \left(\bar{\vartheta}_{3}-\bar{\vartheta}_{4}\right)\right. \\
& \left.+W_{\mathrm{cc}} \sin \left(\bar{\vartheta}_{3}-\bar{\vartheta}_{4}\right)-W_{\mathrm{cc} 13} \sin \left(\bar{\vartheta}_{1}-\bar{\vartheta}_{3}\right)-W_{\mathrm{cc} 23} \sin \left(\bar{\vartheta}_{2}-\bar{\vartheta}_{3}\right)\right] \\
T_{\mathrm{o} 4}= & T_{\mathrm{su}}\left[W_{\mathrm{s} 0}+W_{\mathrm{sc} 14} \cos \left(\bar{\vartheta}_{1}-\bar{\vartheta}_{4}\right)+W_{\mathrm{sc} 24} \cos \left(\bar{\vartheta}_{2}-\bar{\vartheta}_{4}\right)+W_{\mathrm{sc}} \cos \left(\bar{\vartheta}_{3}-\bar{\vartheta}_{4}\right)\right. \\
& \left.-W_{\mathrm{cc}} \sin \left(\bar{\vartheta}_{3}-\bar{\vartheta}_{4}\right)-W_{\mathrm{cc} 14} \sin \left(\bar{\vartheta}_{1}-\bar{\vartheta}_{4}\right)-W_{\mathrm{cc} 24} \sin \left(\bar{\vartheta}_{2}-\bar{\vartheta}_{4}\right)\right] .
\end{aligned}
$$

The right side of each equation in (55) represents the load torque imposed by the vibrating system on each exciter. The first term represents the load torque excited by its own eccentric rotor, and the other terms include the phase difference sine term coupling torques and phase difference cosine 
term coupling torques between this exciter and other three exciters. It is found that the coupling torques of the sine term between any two exciters has the same sign of action on a single motor, so the system uses the coupling torques of the cosine term between the exciters to limit the increase of the speeds of the phase-leading eccentric rotors and to drive the phase-lagging eccentric rotors. The coupling torques between two exciters can be expressed by the product of the maximum value of the corresponding cosine coupling term and the kinetic energy $T_{\mathrm{su}}$.

When cosine coupling terms are not equal to 0 and $\Delta T_{\mathrm{o1} 2}, \Delta T_{\mathrm{o} 34}, \Delta T_{\mathrm{o}}$ are all equal to 0 , the four rotors rotate synchronously. When $\Delta T_{\mathrm{o} 12} \neq 0, \Delta T_{\mathrm{o} 34} \neq 0, \Delta T_{\mathrm{o}} \neq 0$, even if the motor parameters of the two exciters are exactly the same, the output torque of the exciter is a variable that changes with the phase difference between the exciters. The synchronous torque is used to adjust the load torques between the exciters. The synchronous ability coefficients is defined by the ratio of the synchronous torque to the load torque. The load torque between exciters 1 and 2 can be expressed as:

$$
T_{\mathrm{L} 12}= \begin{cases}2 T_{\mathrm{su}}\left(W_{\mathrm{s} 0}+W_{\mathrm{sc}}+2 W_{\mathrm{sc} 13}^{*}\right), & W_{\mathrm{cc}}>0, \\ 2 T_{\mathrm{su}}\left(W_{\mathrm{s} 0}-W_{\mathrm{sc}}\right), & W_{\mathrm{cc}}<0 .\end{cases}
$$

where $W_{\mathrm{sc13}}^{*}$ is the value taken under the calculation result of the phase angle when $W_{\mathrm{cc}}>0$. It can be found that $W_{\mathrm{sc} 13}^{*}=W_{\mathrm{sc} 14}^{*}=W_{\mathrm{sc} 23}^{*}=W_{\mathrm{sc} 24}^{*}$, equation (56) is the simplified result. The maximum value of the cosine line coupling load torque between the exciters 1 and 2 can be known from (25) and expressed as $2 T_{\mathrm{su}}\left|W_{\mathrm{cc}}\right|$. The synchronous ability coefficient between the exciter 1 and $2 \zeta_{12}$ can be expressed as follows. The cosine coupling coefficient in the expression of the synchronous ability coefficient between the exciters 3 and 4 is also $W_{\text {cc }}$, according to the structural symmetry of the system, we have $\zeta_{34}=\zeta_{12}$.

$$
\zeta_{12}= \begin{cases}\frac{W_{\mathrm{cc}}}{W_{\mathrm{s} 0}+W_{\mathrm{sc}}+2 W_{\mathrm{sc} 13}^{*}}, & W_{\mathrm{cc}}>0, \\ \frac{-W_{\mathrm{cc}}}{W_{\mathrm{s} 0}-W_{\mathrm{sc}}}, & W_{\mathrm{cc}}<0 .\end{cases}
$$


Similarly, the load torque between the exciters $1 \& 2$ and the exciters $3 \& 4$ can be expressed as:

$$
\begin{gathered}
T_{\mathrm{L}}= \begin{cases}2 T_{\mathrm{su}}\left(2 W_{\mathrm{s} 0}+2 W_{\mathrm{sc}}+4 W_{\mathrm{sc} 13}^{*}\right), & W_{\mathrm{cc}}>0, \\
2 T_{\mathrm{su}}\left(2 W_{\mathrm{s} 0}-2 W_{\mathrm{sc}}\right), & W_{\mathrm{cc}}<0 .\end{cases} \\
\zeta= \begin{cases}\frac{\left|W_{\mathrm{cc} 13}+W_{\mathrm{cc} 14}+W_{\mathrm{cc} 23}+W_{\mathrm{cc} 24}\right|}{2 W_{\mathrm{s} 0}+2 W_{\mathrm{sc}}+4 W_{\mathrm{sc} 13}^{*}}, & W_{\mathrm{cc}}>0, \\
\frac{\left|W_{\mathrm{cc} 13}+W_{\mathrm{cc} 14}+W_{\mathrm{cc} 23}+W_{\mathrm{cc} 24}\right|}{2 W_{\mathrm{s} 0}+2 W_{\mathrm{sc}}}, & W_{\mathrm{cc}}<0 .\end{cases}
\end{gathered}
$$

The maximum value of (58) is $2 T_{\mathrm{su}}\left|W_{\mathrm{cc} 13}+W_{\mathrm{cc} 14}+W_{\mathrm{cc} 23}+W_{\mathrm{cc} 24}\right|$, then the synchronous ability coefficient between the exciter $1 \& 2$ and $3 \& 4 \zeta$ can be expressed as equation (59).

Through numerical analysis, the relationship between the synchronous ability coefficients and the dimensionless parameters $r_{\mathrm{e}}$ can be obtained as shown in Fig. 6.
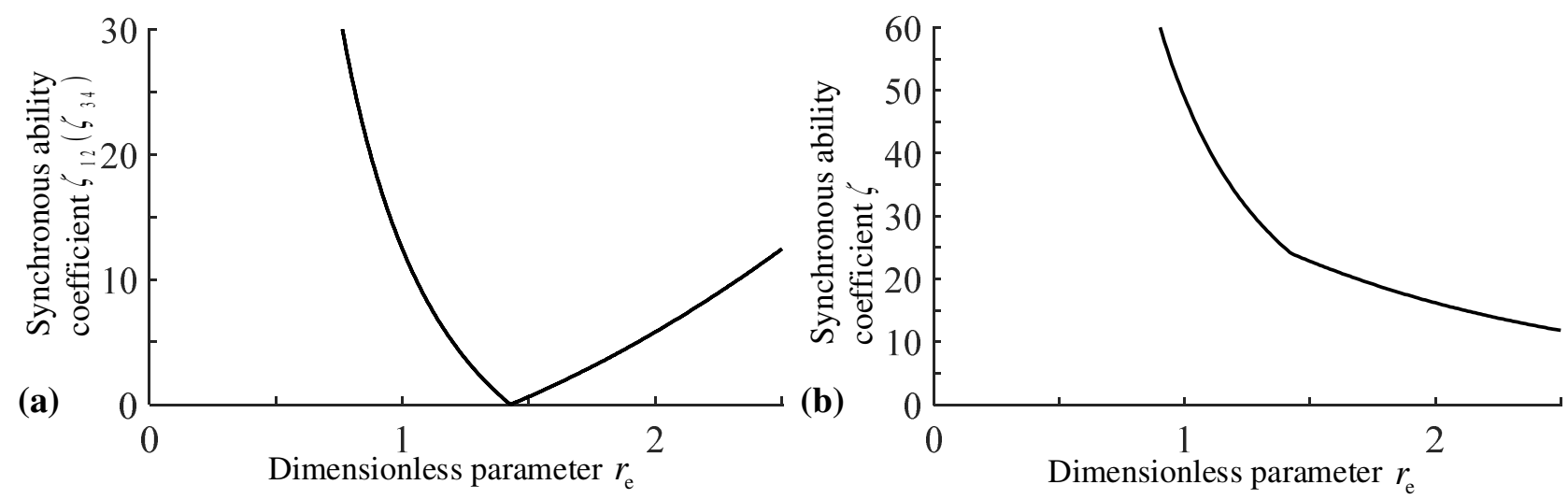

Fig. 6 The synchronous ability coefficients; (a) $\zeta_{12}\left(\zeta_{34}\right)$; (b) $\zeta$

When $r_{\mathrm{e}}=1.414$, we have $\zeta_{12}=0, W_{\mathrm{cc}}=0$. It proves that $r_{\mathrm{e}}=1.414$ is the critical point at which the exciters synchronize. Fig. 6 (a) shows that when the value of $r_{\mathrm{e}}$ deviates from 1.414, the synchronous ability coefficient $\zeta_{12}$ increases rapidly, and under most values of $r_{\mathrm{e}}, \zeta_{12}$ is greater than 1. It shows that when controlling the circular motion of the machine body, and when the two sets of exciters are installed opposite to each other, a good synchronization performance can be achieved. It can be seen from Fig. 6 (b) that the synchronous ability coefficient between the two groups of exciters $\zeta$ shows a trend of gradually decreasing with $r_{\mathrm{e}}$. And at $r_{\mathrm{e}}=1.414, \zeta$ decreases at a slower rate. But values of $\zeta$ are always greater than 10 . 
Considering the phase differences stable interval, good synchronous ability and stability comprehensively, we obtain the parameter determination method of eccentric force offsetting self-synchronization for idle state. In this paper, it can be described as the given value interval $(1.414,2.5)$ of $r_{\mathrm{e}}$. This design can ensure the crusher's actual working conditions, in which the system should be in a self-synchronizing cancellation state and the rigid body, that is, the outer cone should be in the stationary or the slightly vibrating state.

\section{The relationship between crushing force and equivalent stiffness for material crushing state}

After the crusher is filled with materials and enters the full-load state, the equivalent stiffness of the system in all directions is based on the original rubber spring stiffness plus the material's equivalent stiffness. The increase of the equivalent stiffness in each direction also increases the natural frequency. While the operating frequency of the motor remains unchanged, the frequency ratio of the system reduces, so that the system transitions from the zone of far exceeding resonance working to the zone of sub-resonant. Under the synchronization condition in the sub-resonance zone, the frequency ratio of each direction is under 1. Solving nonlinear differential equations (21)-(24) through vibrating parameters to obtain phase differences at full load, $2 \bar{\alpha}_{10}=0,2 \bar{\alpha}_{20}=0,2 \bar{\alpha}_{30}=0$ is obtained. The response of the outer cone of the system in three directions of $x, y, \psi$ is superimposed according to equation (15).

The outer cone and inner cone of the crusher are in contact with the materials through the inner wall and there is a great friction between the inner wall and the materials. Considering that the response of the rigid body in $\psi$ direction is small, so the friction torque between the inner wall and the materials can offset the response in $\psi$ direction. The system motion form is a circular motion with the response superimposed in $x$ and $y$ directions. Fig. 7 shows the dynamic model of the plane motion of the outer cone in $x$ direction when it is fully loaded. The dynamic model in $y$ direction is 
equivalent to that in $x$ direction.

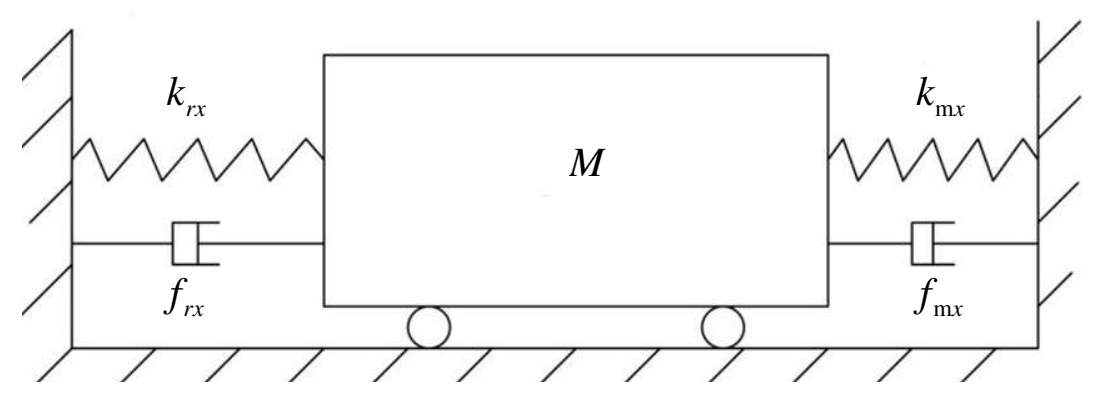

Fig. 7 Simplified linear vibration dynamic model of outer cone for material crushing state

When the crusher is full of materials, the system runs synchronously with four eccentric rotors in zero phases, which belongs to the self-driving vibration. The compressed materials can be regarded as a spring, and its equivalent damping is $f_{\mathrm{m} x}$; Suppose the statistical characteristics of the compressed materials is isotropic, and its equivalent stiffness is the same in all directions, we set its extrusion stiffness to $\delta$ times the total weight of the moving rigid body, that is:

$$
k_{\mathrm{m} x}=k_{\mathrm{m} y}=\delta M g
$$

where $\delta$ is the stiffness coefficient of the materials. The equivalent stiffness and equivalent damping of linear vibration are respectively:

$$
\begin{aligned}
& k_{x}=k_{r x}+k_{\mathrm{m} x}, \\
& f_{x}=f_{r x}+f_{\mathrm{m} x} .
\end{aligned}
$$

\subsection{Working amplitude and crushing force at full load}

The differential equation of motion of the four-vibrator self-driving vibration in the $x$ direction can be expressed as:

$$
M\left(f_{r x}+f_{\mathrm{m} x}\right) \&+\left(k_{r x}+k_{\mathrm{m} x}\right) x=4 m_{0} r \omega_{\mathrm{m} 0}^{2} \cos \left(\omega_{\mathrm{m} 0} t\right)
$$

that is:

$$
2 \xi_{\mathrm{xm}} \omega_{\mathrm{mnx}} \& \omega_{\mathrm{mnx}}^{2} x=4 r r_{\mathrm{m}} \omega_{\mathrm{m} 0}^{2} \cos \left(\omega_{\mathrm{m} 0} t\right) \text {. }
$$

where

$$
\omega_{\mathrm{mn} x}=\sqrt{\frac{k_{r x}+k_{\mathrm{m} x}}{M}}, \quad \xi_{x \mathrm{~m}}=\frac{f_{r x}+f_{\mathrm{m} x}}{2 \sqrt{\left(k_{r x}+k_{\mathrm{m} x}\right) M}} .
$$

From this, the responses and response speeds of the full-load circular motion of the system can be 
obtained as:

$$
\begin{aligned}
& x=4 r r_{\mathrm{m}} \mu_{x} \cos \left(\omega_{\mathrm{m} 0} t-\gamma_{x}\right), \\
& y=4 r r_{\mathrm{m}} \mu_{y} \sin \left(\omega_{\mathrm{m} 0} t-\gamma_{y}\right), \\
& \&=-4 r r_{\mathrm{m}} \omega_{\mathrm{m} 0} \mu_{x} \sin \left(\omega_{\mathrm{m} 0} t-\gamma_{x}\right), \\
& \&=4 r r_{\mathrm{m}} \omega_{\mathrm{m} 0} \mu_{y} \cos \left(\omega_{\mathrm{m} 0} t-\gamma_{y}\right) .
\end{aligned}
$$

The overall restoring forces of the system are:

$$
\begin{aligned}
F_{x} & =\left(k_{\mathrm{m} x}+k_{r x}\right) x+\left(f_{r x}+f_{\mathrm{m} x}\right) \& \& \\
& =4 r r_{\mathrm{m}} \mu_{x}\left[\left(k_{r x}+k_{\mathrm{m} x}\right) \cos \left(\omega_{\mathrm{m} 0} t-\gamma_{x}\right)-\left(f_{r x}+f_{\mathrm{m} x}\right) \omega_{\mathrm{m} 0} \sin \left(\omega_{\mathrm{m} 0} t-\gamma_{x}\right)\right] . \\
F_{y} & =\left(k_{r y}+k_{\mathrm{m} y}\right) y+\left(f_{r y}+f_{m y}\right) \& \\
& =4 r r_{\mathrm{m}} \mu_{y}\left[\left(k_{r y}+k_{\mathrm{m} y}\right) \sin \left(\omega_{\mathrm{m} 0} t-\gamma_{y}\right)+\left(f_{r y}+f_{\mathrm{m} y}\right) \omega_{\mathrm{m} 0} \cos \left(\omega_{\mathrm{m} 0} t-\gamma_{y}\right)\right] .
\end{aligned}
$$

From Fig. 7, the forces between the outer cone and the material are:

$$
\begin{aligned}
F_{\mathrm{m} x} & =k_{\mathrm{m} x} x+f_{\mathrm{m} x} \& \& \\
& =4 r r_{\mathrm{m}} \mu_{x}\left[k_{\mathrm{m} x} \cos \left(\omega_{\mathrm{m} 0} t-\gamma_{x}\right)-f_{\mathrm{m} x} \omega_{\mathrm{m} 0} \sin \left(\omega_{\mathrm{m} 0} t-\gamma_{x}\right)\right] . \\
F_{\mathrm{m} y} & =k_{\mathrm{m} y} y+f_{\mathrm{m} y} \& \\
& =4 r r_{\mathrm{m}} \mu_{y}\left[k_{\mathrm{m} y} \sin \left(\omega_{\mathrm{m} 0} t-\gamma_{y}\right)+f_{\mathrm{m} y} \omega_{\mathrm{m} 0} \cos \left(\omega_{\mathrm{m} 0} t-\gamma_{y}\right)\right] .
\end{aligned}
$$

\subsection{Critical stiffness of crushing materials}

The cone crusher adopts two support schemes, which are supported by rubber springs and plane motion bearings between the outer cone and the machine base. In the static state, the rubber springs and plane motion bearings jointly bear the total weight of the outer cone rigid body $M g$. In the working process, the extrusion force between the outer cone and the materials produces a downward component, and this part of the force is borne by the plane support bearings. Suppose the proportion of the plane bearings carry the weight $M g$ is $\eta, 0<\eta<1$, the rest is carried by the rubber support springs. Suppose the static deformation of the rubber springs in the vertical direction of vibration is $\Delta z$, their vertical stiffness is:

$$
k_{r z}=\frac{M g(1-\eta)}{\Delta z} .
$$

Suppose the ratio of the vertical stiffness of the rubber springs to the shear stiffness is $\kappa$, then the horizontal stiffness is: 


$$
k_{r x}=k_{r y}=\frac{k_{r z}}{\kappa}=\frac{M g(1-\eta)}{\kappa \Delta z} .
$$

Substituting equations (71) and (60) into equation (61), we have:

$$
k_{x}=k_{y}=\left(\frac{1-\eta}{\kappa \Delta z}+\delta\right) M g
$$

then

$$
\omega_{m n x}=\omega_{m n y}=\sqrt{\left(\frac{1-\eta}{\kappa \Delta z}+\delta\right) g} .
$$

The system works in the sub-resonant zone under full-load condition to meet:

$$
\omega_{\mathrm{mn} x}=\omega_{\mathrm{mny}}>\omega_{\mathrm{m} 0} .
$$

Substituting $\omega_{m 0}=104.2 \mathrm{rad} / \mathrm{s}$ and equation (73) into equation (74), we obtain:

$$
\delta>\delta_{\mathrm{c}}=\frac{104 \cdot 2^{2}}{g}-\frac{1-\eta}{\kappa \Delta z} .
$$

where $\delta_{c}$ is called the critical equivalent stiffness coefficient of the crusher's crushing materials.

Fig. 8 shows the influence of system design parameters on the critical stiffness of the crushing materials, including the bearing support coefficient $\eta$, the stiffness ratio of the rubber springs $\kappa$ and the initial vertical deformation of the rubber springs $\Delta z$.
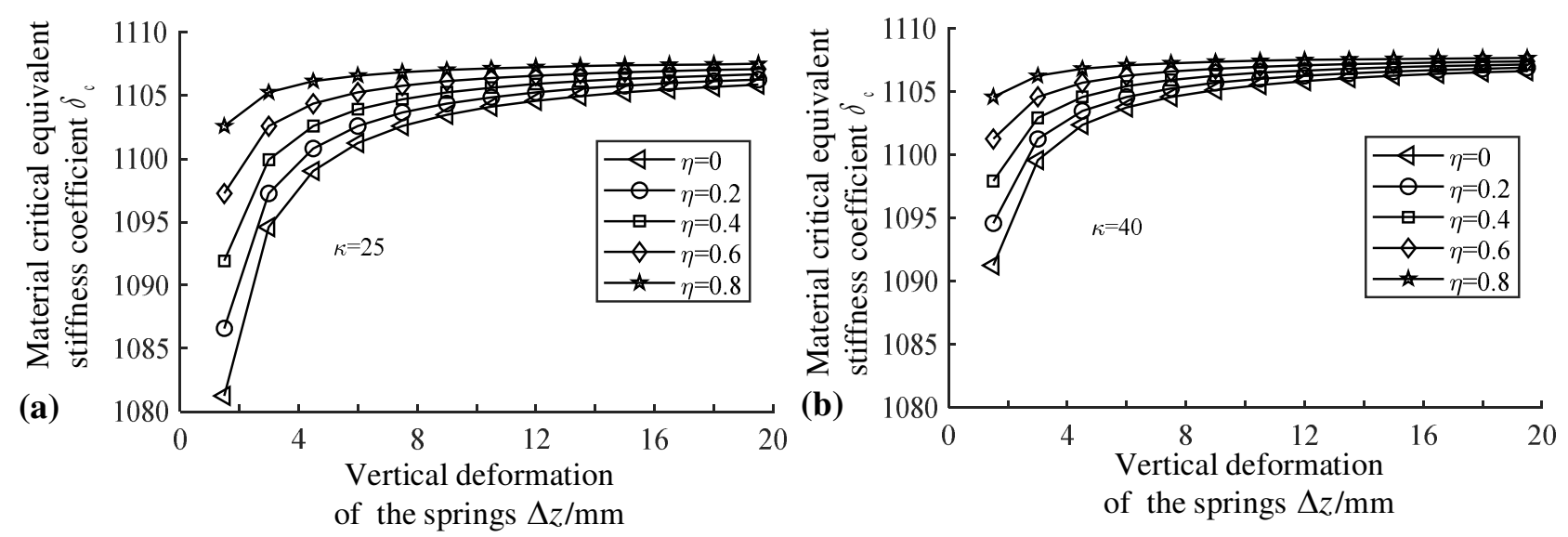

Fig. 8 The influence of system design parameters on the critical equivalent stiffness of crushing materials;

(a) $\kappa=25$; (b) $\kappa=40$.

It can be seen that as $\Delta z$ increases, the critical equivalent stiffness coefficient of the materials $\delta_{\mathrm{c}}$ increases; With the increase of $\eta$ and $\kappa$, the increase of $\delta_{\mathrm{c}}$ slows down and gradually tends to a stable value, and the difference between the (a) values and (b) values are not large, indicating that 
the influence of $\eta$ and $\kappa$ on $\delta_{\mathrm{c}}$ can be ignored.
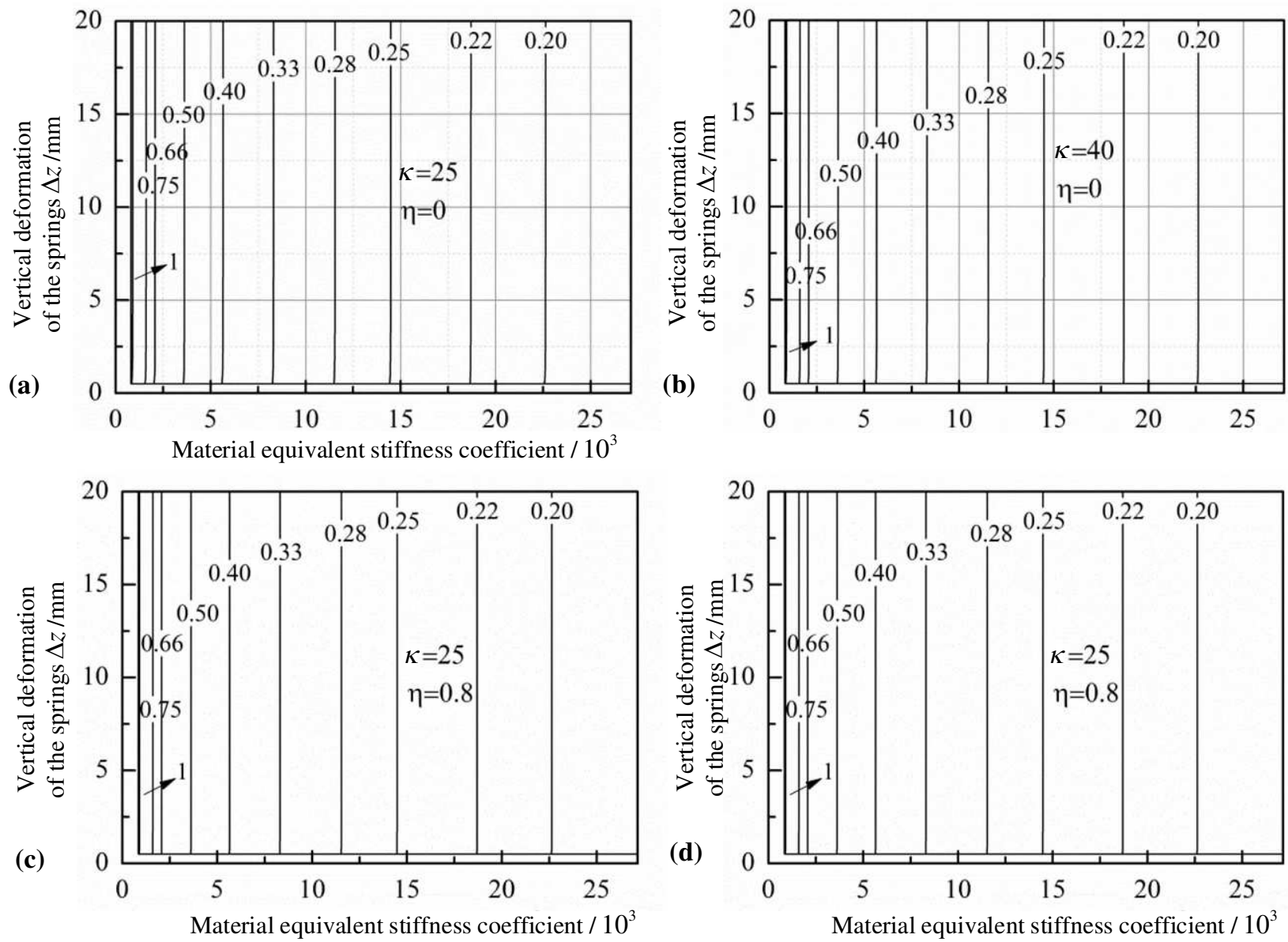

Fig. 9 The effect of material equivalent stiffness and spring deformation on the frequency ratio of the of the operating point; (a) $\kappa=25, \eta=0$; (b) $\kappa=40, \eta=0$; (c) $\kappa=25, \eta=0.8$; (d) $\kappa=40, \eta=0.8$.

In order to explore the influence of the material stiffness coefficient and system design parameters on the frequency ratio of the operating point, the stiffness ratio of the rubber springs $\kappa$ are taken as 25 and 40 respectively, and the bearing support coefficient $\eta$ are taken as 0 and 0.8 respectively. The corresponding change rule is shown in Fig. 9. It can be seen from the comparison of Fig. 9 (a) -(d) that the change of $\eta$ and $\kappa$ of the crusher has little effect on the frequency ratio of the operating point, especially when the operating point frequency is relatively small. In Fig. 9, the frequency ratio contour of the working point changes the fastest in the direction of the change of the material equivalent stiffness coefficient, indicating that the frequency of the working point mainly depends on the equivalent stiffness coefficient of the material.

According to the analysis of the parameter value interval in Fig. 10, the change speed and amplitude 
of the operating point frequency ratio $\omega_{\mathrm{m} 0} / \omega_{\mathrm{n} x}$ in the vertical deformation $\Delta z$ direction of the spring are smaller than the change speed and amplitude in the direction of the material equivalent stiffness. Combining the data of spring deformation $\Delta z=1.5 \mathrm{~mm}$ in Table 1 and Fig. 10, it can be known that the system must work in the sub-resonance zone to ensure that the operating frequency of the system is less than the natural frequency under full-load condition, and the value of the material equivalent stiffness coefficient must not less than 1100 , which is numerically consistent with the material critical equivalent stiffness coefficient in Fig. 8.

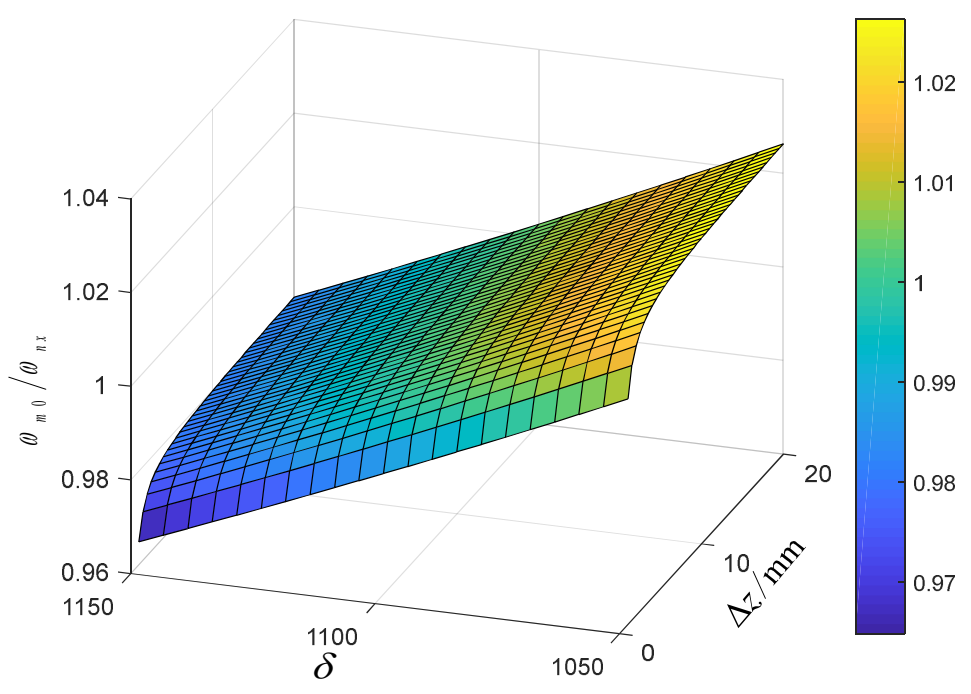

Fig. 10 The parameter value range to ensure the system works in the sub-resonance zone when $\kappa=25, \eta=0$.

Tab. 1 The relationship between rubber spring parameters and supporting load coefficient and working frequency ratio under full-load condition $(\Delta z=1.5 \mathrm{~mm})$

\begin{tabular}{cccccc}
\hline \multirow{2}{*}{$\kappa$} & \multicolumn{5}{c}{ material equivalent stiffness coefficient/1033 } \\
& & 1 & 1.05 & 1.1 & 1.15 \\
\hline \multirow{2}{*}{25} & 0 & 1.0388 & 1.0144 & 0.9916 & 0.9703 \\
& 0.8 & 1.0498 & 1.0246 & 1.0011 & 0.9793 \\
40 & 0 & 1.0439 & 1.0192 & 0.9961 & 0.9745 \\
& 0.8 & 1.0508 & 1.0256 & 1.0021 & 0.9801 \\
\hline
\end{tabular}

\subsection{Material effective crushing coefficient and discharge port compression coefficient}

For the vibrating system shown in Fig. 7, the critical damping is:

$$
f_{\mathrm{c}}=2 \sqrt{M\left(k_{r x}+k_{\mathrm{m} x}\right)} .
$$

Set the damping coefficient of the spring and material in $x$ direction as: 


$$
c_{r x}=\frac{f_{r x}}{2 M}, \quad c_{\mathrm{m} x}=\frac{f_{\mathrm{m} x}}{2 M} .
$$

Substituting (76) and (77) into (66) and (67), we have:

$$
\begin{aligned}
& F_{x}=4 r m_{0} \mu_{x}\left[\left(\frac{1-\eta}{\kappa \Delta z}+\delta\right) g \cos \left(\omega_{\mathrm{m} 0} t-\gamma_{x}\right)-2 \omega_{\mathrm{m} 0}\left(c_{r x}+c_{\mathrm{m} x}\right) \sin \left(\omega_{\mathrm{m} 0} t-\gamma_{x}\right)\right], \\
& F_{y}=4 r m_{0} \mu_{y}\left[\left(\frac{1-\eta}{\kappa \Delta z}+\delta\right) g \sin \left(\omega_{\mathrm{m} 0} t-\gamma_{y}\right)+2 \omega_{\mathrm{m} 0}\left(c_{\mathrm{r} y}+c_{\mathrm{m} y}\right) \cos \left(\omega_{\mathrm{m} 0} t-\gamma_{y}\right)\right] .
\end{aligned}
$$

The forces between the outer cone and the material are:

$$
\begin{aligned}
& F_{\mathrm{m} x}=4 r r_{\mathrm{m}} \mu_{x}\left[\delta g \cos \left(\omega_{\mathrm{m} 0} t-\gamma_{x}\right)-2 \omega_{\mathrm{m} 0} c_{\mathrm{m} x} \sin \left(\omega_{\mathrm{m} 0} t-\gamma_{x}\right)\right], \\
& F_{\mathrm{m} y}=4 r r_{\mathrm{m}} \mu_{y}\left[\delta g \sin \left(\omega_{\mathrm{m} 0} t-\gamma_{y}\right)+2 \omega_{\mathrm{m} 0} c_{\mathrm{m} x} \cos \left(\omega_{\mathrm{m} 0} t-\gamma_{y}\right)\right] .
\end{aligned}
$$

Then the magnitude of the restoring force of circular motion is:

$$
F_{s}=4 r m_{0} \mu_{x} \sqrt{\omega_{\mathrm{nm} x}^{4}+4 \omega_{\mathrm{m} 0}^{2}\left(c_{\mathrm{r} x}+c_{\mathrm{m} x}\right)^{2}} .
$$

The magnitude of the force between the outer cone and the materials is:

$$
F_{\mathrm{m}}=4 r m_{0} \mu_{x} \sqrt{\delta^{2} g^{2}+4 \omega_{\mathrm{m} 0}^{2} c_{\mathrm{m} x}^{2}} .
$$

The ratio of restoring force to the excitation is defined as the system restoring force coefficient $\lambda_{\mathrm{s}}$ :

$$
\lambda_{\mathrm{s}}=\frac{F_{\mathrm{s}}}{4 r m_{0} \omega_{\mathrm{m} 0}^{2}}=\mu_{x} \sqrt{n_{x \mathrm{~m}}^{-4}+\left(2 \xi_{x} n_{x \mathrm{~m}}^{-1}\right)^{2}} .
$$

The ratio of the force between the outer cone and the materials to the excitation is defined as the crushing force coefficient $\lambda_{\mathrm{m}}$ :

$$
\lambda_{\mathrm{m}}=\frac{F_{\mathrm{m}}}{4 r m_{0} \omega_{\mathrm{m} 0}^{2}}=\mu_{x} \sqrt{\left(\frac{\delta g}{\omega_{\mathrm{m} 0}^{2}}\right)^{2}+\left(\frac{2 c_{\mathrm{m} x}}{\omega_{\mathrm{m} 0}}\right)^{2}} .
$$

The ratio $\lambda_{\mathrm{s}}$ to $\lambda_{\mathrm{m}}$ is defined as the effective crushing coefficient of the crusher. Higher crushing efficiency and better crushing effect needs larger effective crushing coefficient.

$$
\sigma=\frac{\lambda_{\mathrm{m}}}{\lambda_{\mathrm{s}}}
$$

According to equation (64), the radial displacement of the outer cone during the working process can be calculated, and the compression coefficient of the discharge port can be obtained by the ratio of the circular radial movement to the nominal amplitude as:

$$
\rho_{\mathrm{s}}=\frac{\sqrt{x^{2}+y^{2}}}{4 r r_{\mathrm{m}}}=\sqrt{2} \mu_{x} .
$$


Discuss the material equivalent stiffness coefficient $\delta$ and the system damping ratio $\xi_{x}$ to the above coefficients. Fig. 11 and Fig. 12 respectively show the change laws of three sets of force coefficient values and the compression coefficient of the discharge port with the stiffness coefficient of the materials.
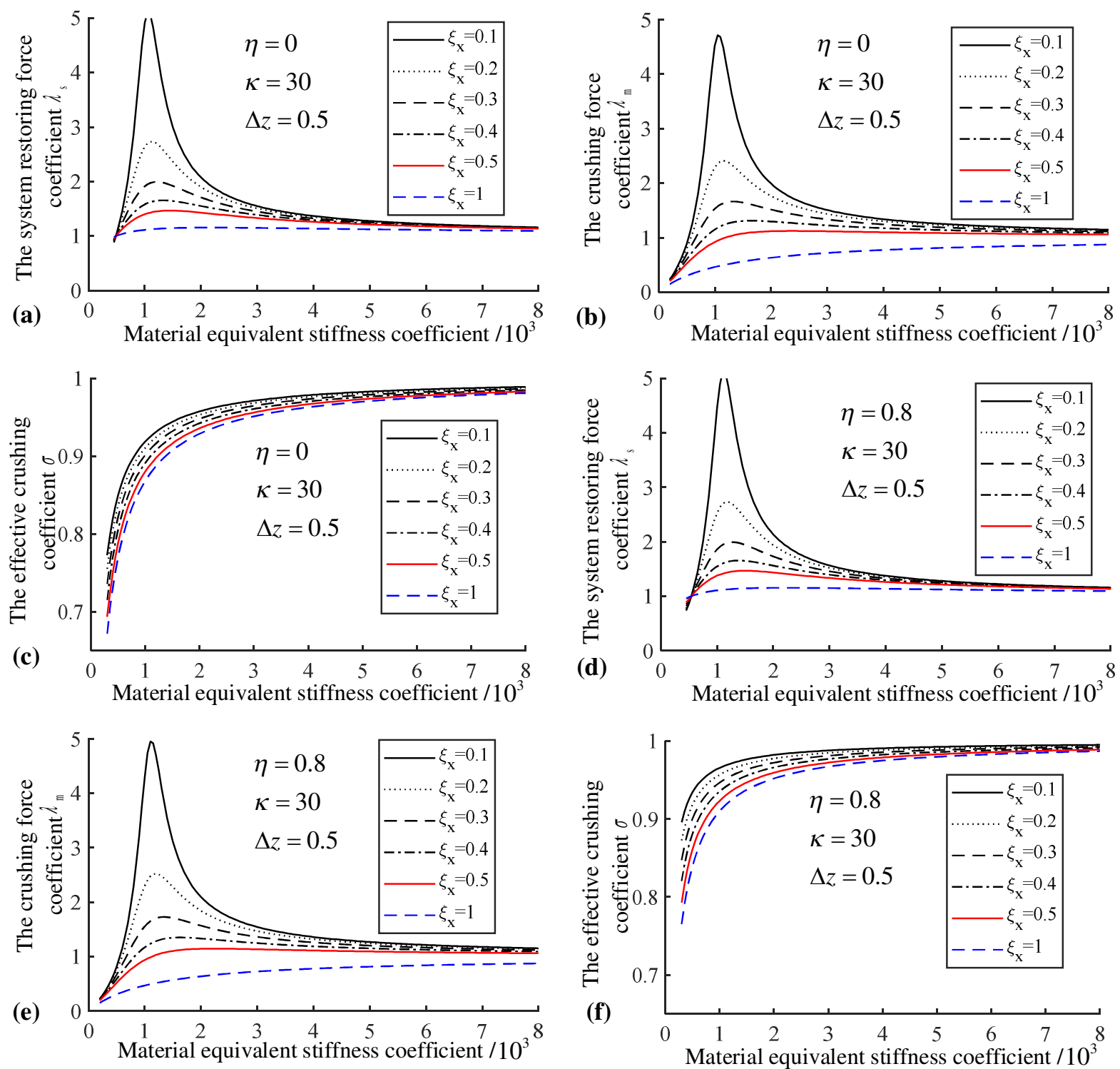

Fig. 11 The effect of equivalent stiffness coefficient and system damping ratio on crusher's crushing force coefficient and effective crushing force coefficient; (a) The system restoring force coefficient when $\kappa=30$, $\eta=0, \Delta z=0.5$; (b) The crushing force coefficient when $\kappa=30, \eta=0, \Delta z=0.5$; (c) The effective crushing coefficient when $\kappa=30, \eta=0, \Delta z=0.5$; (d) The system restoring force coefficient when $\kappa=30$, $\eta=0.8, \Delta z=0.5$; (e) The crushing force coefficient when $\kappa=30, \eta=0.8, \Delta z=0.5$; (f) The effective crushing coefficient when $\kappa=30, \eta=0.8, \Delta z=0.5$.

Take values of $\kappa=30, \Delta z=0.5 \mathrm{~mm}, \eta=0$ and 0.8 respectively. Set the system damping ratio under full-load to $0.1,0.2,0.3,0.4,0.5$ and 1 respectively. By comparing the first three sets of data with 
the last three sets of data, it can be found that the change in $\eta$ has little effect on the values of the force coefficients and can be ignored. The three groups of force coefficient values all decrease with the increase of the system damping ratio $\xi_{x}$. This effect is more obvious for the restoring force coefficient and the crushing force coefficient, but has no obvious effect on the value of the effective breaking force coefficient.

The restoring force coefficient and the crushing force coefficient show a trend of first increasing and then decreasing with the addition of materials. When the equivalent stiffness coefficient of the material breaks through about 1100, the system enters the sub-resonance zone and gradually stabilizes from the maximum. The effective crushing coefficient gradually increases with the addition of materials and gradually reaches the steady state maximum when the system enters the sub-resonance zone.

From equation (87), we can see that $\eta, \kappa, \Delta z$ are much less than $\delta$. Also it can be seen from Fig. 12 that as the system damping ratio increases, the compression coefficient of the discharge port decreases; And with the addition of materials, the compression coefficient gradually decreases when the operating point moves to the sub-resonance zone.

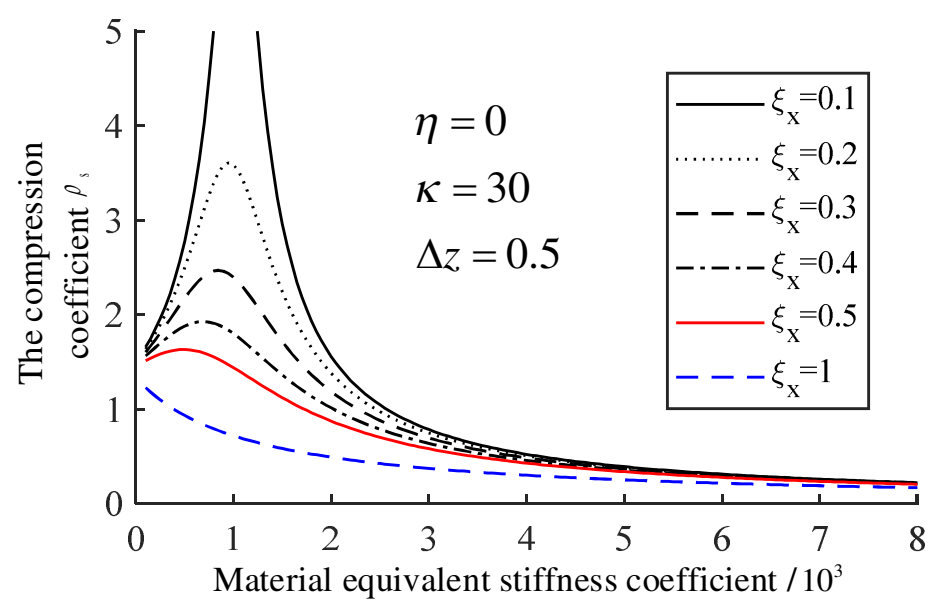

Fig. 12 The effect of equivalent stiffness coefficient and system damping ratio on compression coefficient The V-shaped space between the outer cone and the fixed cone periodically changes in amplitude and the amplitude decreases. At this time, the smaller and finer crushed particles will be obtained. It 
is necessary to adjust the material equivalent stiffness coefficient $\delta$ to adjust the actual working frequency, which is at the sub-resonance zone critical point, to meet the crushing requirements according to specific engineering practice and crushing particle size requirements.

\section{Computer simulation}

In order to prove the correctness of the above theoretical research results, the computer simulation method is used for vibrating analysis. The digital simulation is based on the principle of numerical calculation. In this paper, we use Kutta-Runge theory and $\mathrm{C}$ language to compile a simulation program to analyze the synchronous speeds, steady-state phase differences, and displacement responses in each direction of the plane motion.

\subsection{Idle state analysis}

The quality of the outer cone $m=1260 \mathrm{~kg}$ and the mass of the eccentric rotor of the exciter $m_{0}=$ $60 \mathrm{~kg}$ are given as above. For known parameter $R=1.6 \mathrm{~m}, M=1500 \mathrm{~kg}, J_{\mathrm{w}}=1088 \mathrm{~kg} \mathrm{~m}^{2}$, the dimensionless coupling parameter $r_{\mathrm{e}}=1.5$ can be calculated, which meets the above calculation. Subsequent simulations are carried out under the condition of $r_{\mathrm{e}}=1.50$.

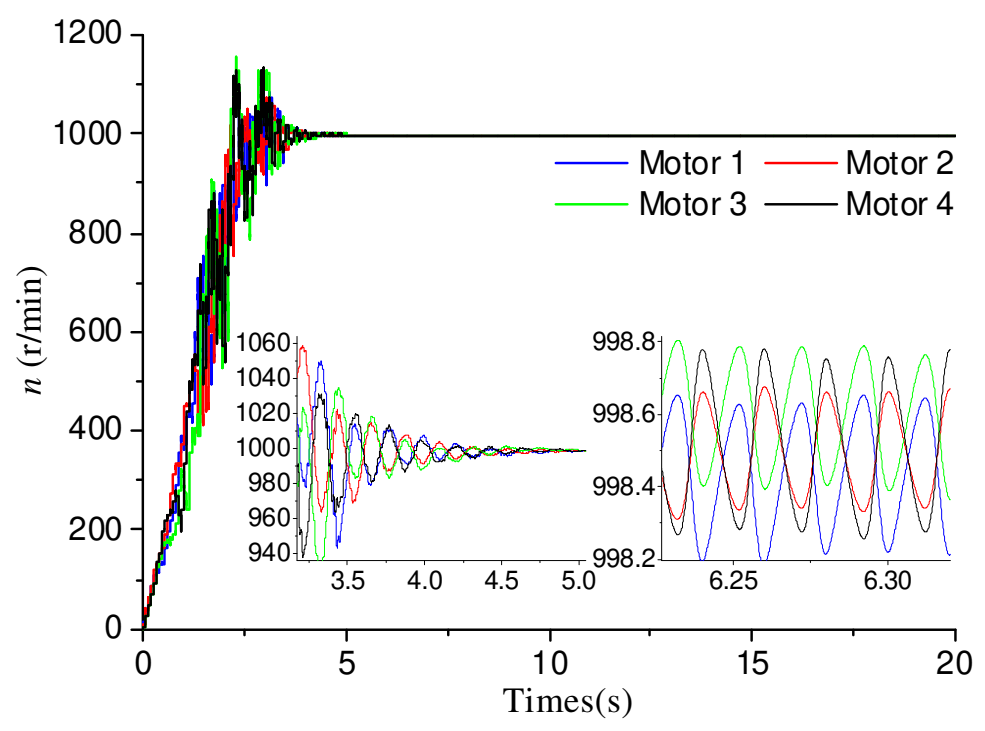

Fig. 13 Synchronous speeds of four motors

The computer simulation analysis results of the four-machine self-synchronous speeds are shown in 
Fig. 13. Since the four motors are supplied the same frequency power, so the four exciters driven by the four motors are also the same. The steady-state speeds of the four motors tend to be the same, and the accelerations are also equal. As shown in Fig. 13, the speeds of the four motors began to converge at $4 \mathrm{~s}$, and were in a fluctuating state before then. After $5 \mathrm{~s}$, the synchronous torque between the four motors makes the speeds of the four motors start to be in a stable state. The final synchronous operating speed is at around $998.5 \mathrm{r} / \mathrm{min}$.

(a)

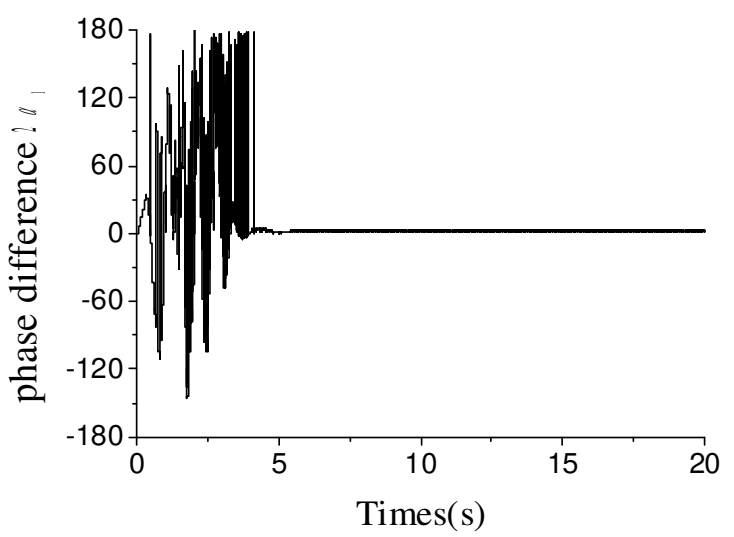

(b)

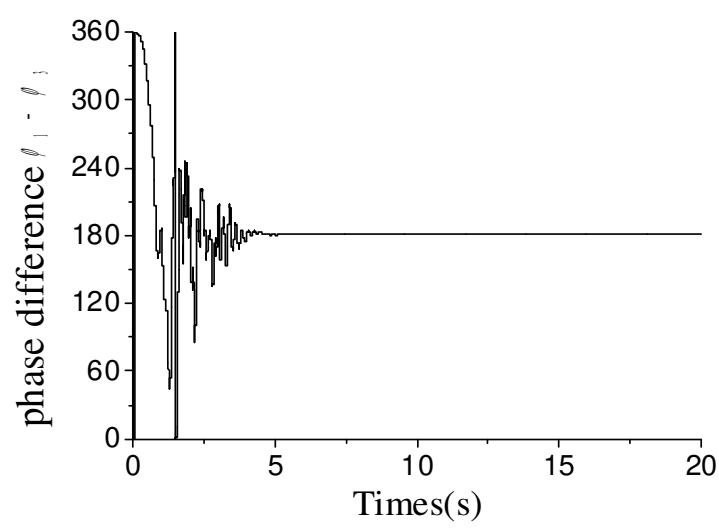

(c)

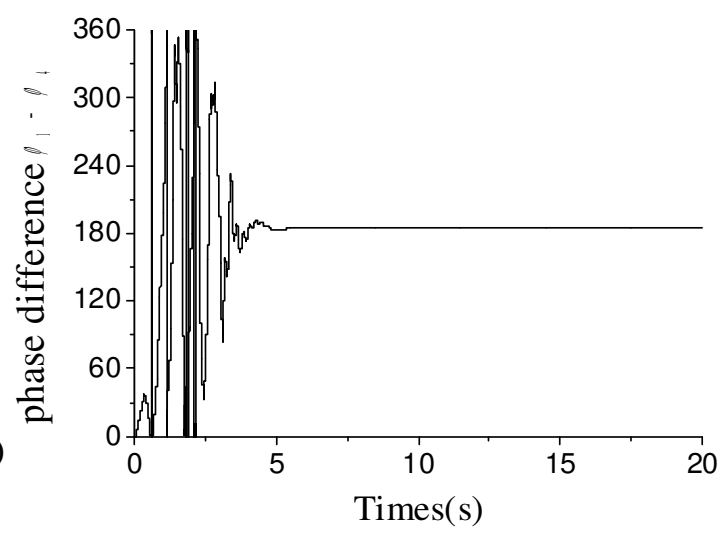

Fig. 14 Phase differences between URs; (a) Phase difference between exciter 1 and 2; (b) Phase difference between exciter 1 and 3; (c) Phase difference between exciter 1 and 4

$2 \alpha_{1}, 2 \alpha_{2}, 2 \alpha_{3}$ are used to adjust the output torques of the motors. The computer simulation selects the phase differences between exciters 1 and 2, exciters 1 and 3, and exciters 1 and 4 . The results are shown in Fig. 14. It can be seen from the figure that the phase difference between exciters 1 and 2 tends to reach a stable state $2 \alpha_{1}=0$ after 5 s. So do the phase differences between exciters 1 and 3, 1 and 4 plotted in Fig. 14 (b) and (c). $\varphi_{1}-\varphi_{3}$ is stable around $181.2^{\circ}$ and $\varphi_{1}-\varphi_{4}$ is around the value of $184.7^{\circ}$. This steady-state value ensures that the two sets of vibrating exciters 
installed in a straight line run synchronously in phase and the two sets of vibrating exciters installed at right angles run synchronously in anti-phase.

The vibrating responses in all directions of the system cancel each other out and the body is in a state of no movement or slight vibration. It can be seen from Fig. 15 of the displacement in each direction: the displacement in the $x$ direction is finally stabilized at about $0.13 \mathrm{~mm}$, the displacement in the $y$ direction is finally stabilized at about $0.11 \mathrm{~mm}$, are all close to 0 . And the displacement in the $\psi$ direction is finally stabilized at $2.55^{\circ}$.

(a)

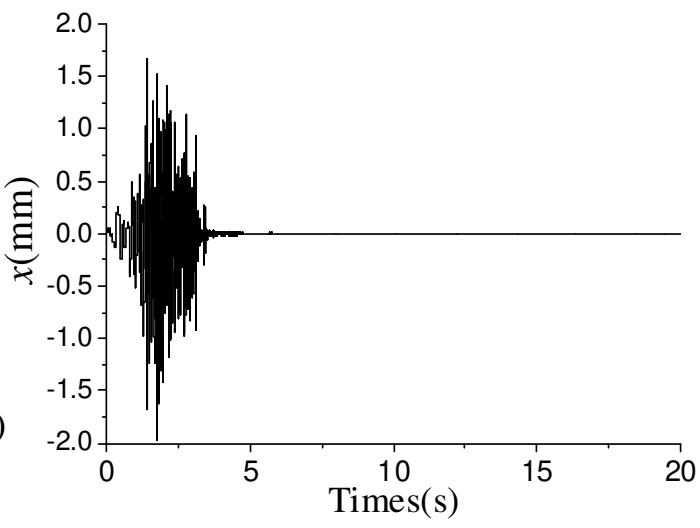

(c)

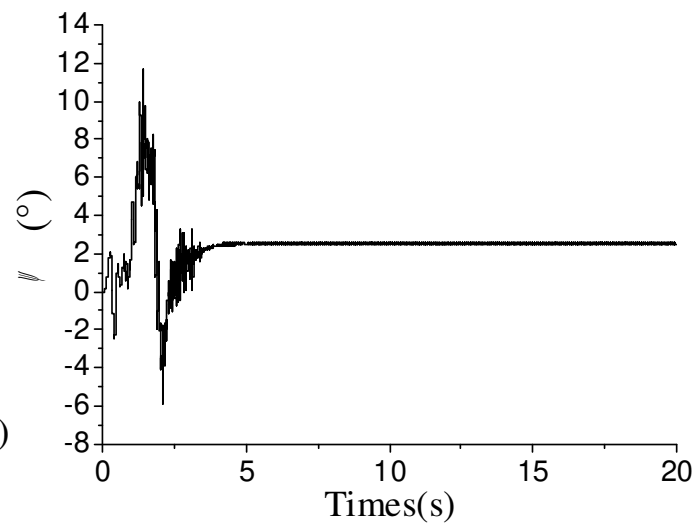

(b)

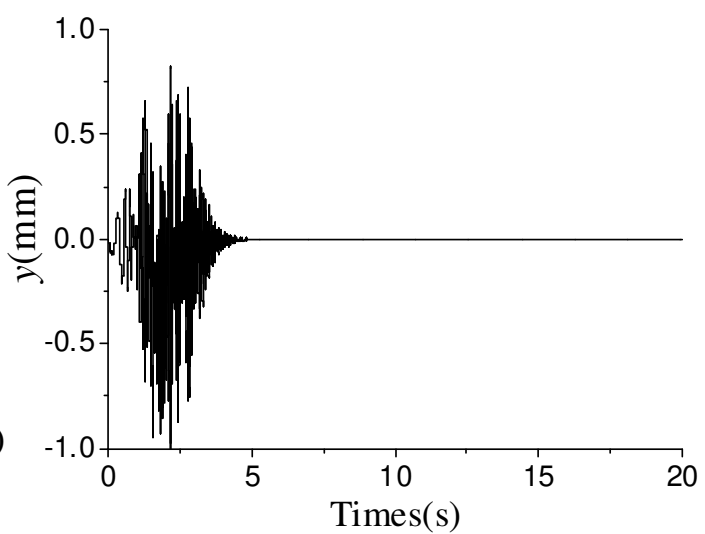

Fig. 15 Stimulus response for idle state in three directions; (a) $x$-direction displacement; (b) $y$-direction displacement; (c) $\psi$-direction displacement

The displacements in all directions for idle state are stable near 0 . The simulation results are basically consistent with the numerical analysis results. The four motors under no-load condition have reached the vibrating synchronous transmission state, that is, the natural frequency of the system is increased by adding the material stiffness to adjust the operating frequency point of the 
system. The speeds of the four motors will reach the steady state again in a short period of time, and the system will be stable for the second time. The state of the body changes the motion form of the body through the change of the phase differences.

\subsection{Full-load simulation analysis}

Corresponding to the idle state, the material crushing state is another critical state of the crusher system. The phase differences and displacements of the critical state after the crusher is full of materials are specifically analyzed.

(a)

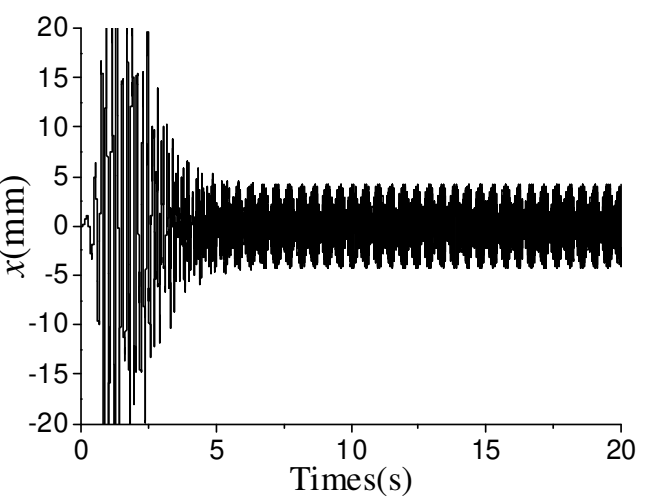

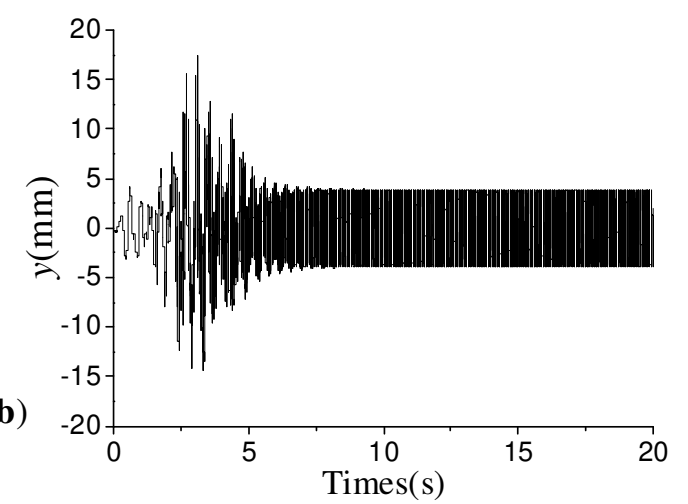

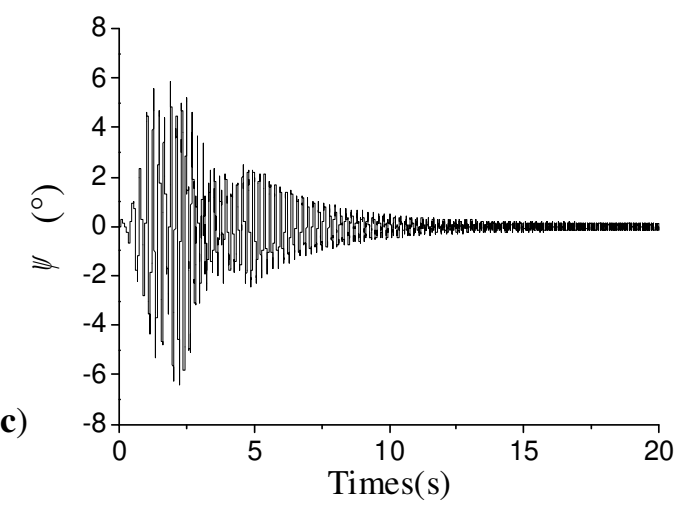

Fig. 16 Stimulus response under full-load condition in three directions; (a) $x$-direction displacement; (b) $y$-direction displacement; (c) $\psi$-direction displacement

Take the damping in the straight line direction of the critical state $f_{x}=f_{y}=5.5 \mathrm{kN} \mathrm{s} / \mathrm{m}$. The rotational damping due to the large rotational friction in the working cavity, should be much larger than that in the straight line direction, we take $f_{\psi}=100 \mathrm{kN} \mathrm{s} / \mathrm{rad}$. The total stiffness in the straight line direction is taken $k_{x}=k_{y}=3 \times 10^{4} \mathrm{kN} / \mathrm{m}$ and the rotational stiffness $k_{\psi}=1.3 \mathrm{kN} \mathrm{m} / \mathrm{rad}$. The synchronous speeds are only related to the damping coefficient of the motor shaft and the 
steady-state phase difference, so the synchronous speed under full-load condition does not change much, as shown in Fig. 13, which is maintained at around $104.2 \mathrm{rad} / \mathrm{s}$.

At this time, the phases between the four motors all tend to be the same to make the linear responses superimpose. The excitation responses are shown in Fig. 16. The amplitudes in the $x$ direction and $y$ direction are approximately equal at around $3.5 \mathrm{~mm}$. In the $\psi$ direction, because the rotation damping is large and the value of the rotation stiffness is small, the rotation angle gradually approaches 0 with time. In this state, the crusher realizes the selection of elliptical motion.

\subsection{Experimental tests and verifications}

To examine the correctness of our theory, numerical and simulation results, we carried out some experimental tests and verifications in this section. The point that the real body of the vibrating cone crusher is hard to be manufactured and fabricated, because of its enormous size (more than 3 meters in length), should be noticed. We built a model (1:3) in the laboratory for a series of vibrating tests. The experimental devices are the experimental cone crusher model with four URs driven by four identical motors (the type and specification is $\mathrm{Y} 2-225 \mathrm{M}-6,380 \mathrm{~V}, 50 \mathrm{~Hz}, 6$-pole, $\Delta$-connection, rated power $7.5 \mathrm{~kW}$, rated speed $980 \mathrm{r} / \mathrm{min}$ ) shown in Fig. 17 (a), a series of testing instruments shown in Fig. 17(b): the acceleration sensors (INV-B-M-10) to acquire accelerations in $x$-, $y$ - and $\psi$-directions, the Hall-sensors (NJK-5002C) to acquire phase differences between URs and the processing analyzer to obtain the results in the form of data. And the parameters of the vibrating cone crusher's experimental model are shown in Tab. 2.

Due to the limitation of experimental conditions, we only carried out steady-state operation experiments under idle state. The experimental results under dimensionless parameter $r_{\mathrm{e}}=1.50$ are illustrated in Fig. 18 and Fig. 19. As we can see from displacement data charts, the displacements in $x$-, $y$ - and $\psi$ - directions are in a state of unstable fluctuation at the beginning. 


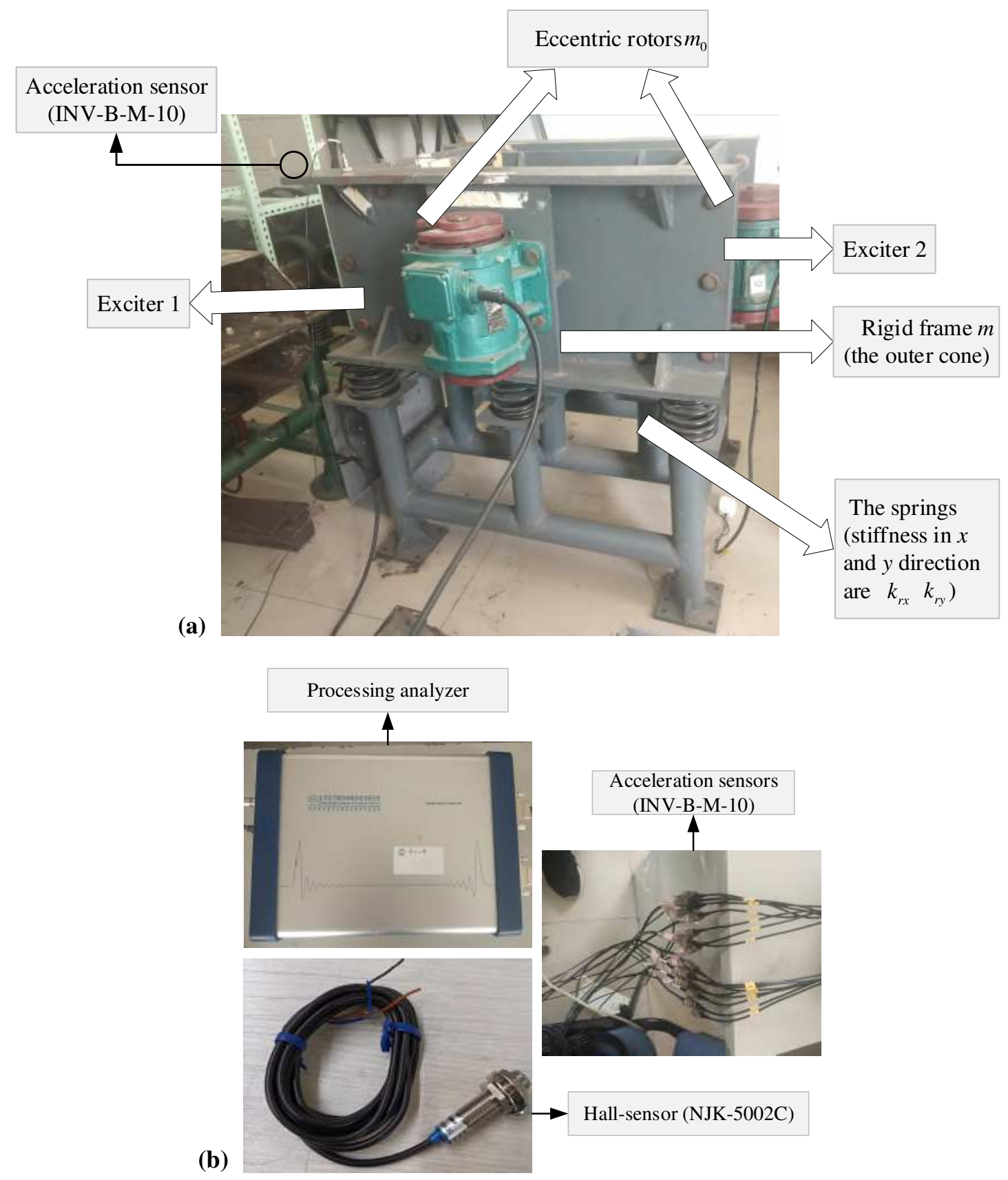

Fig. 17 Experimental facilities; (a) experimental cone crusher model with four URs $\left(r_{\mathrm{e}}=1.50\right)$; (b) a series of testing instruments;

Tab. 2

Parameters of the vibrating cone crusher's experimental model

\begin{tabular}{ccccccccc}
\hline Parameters & $m / \mathrm{kg}$ & $m_{0} / \mathrm{kg}$ & $r_{\mathrm{m}}$ & $r / \mathrm{m}$ & $r_{\mathrm{e}}$ & $J_{\mathrm{w}} / \mathrm{kg} \mathrm{m}^{2}$ & $k_{r x} / \mathrm{kN} / \mathrm{m}$ & $f_{r x} / \mathrm{kN} \mathrm{s} / \mathrm{m}$ \\
\hline Values & 630 & 30 & 0.04 & 0.07 & 1.50 & 57.6 & 135.2 & 18.9 \\
\hline
\end{tabular}

After around 9 seconds, the experimental data of phase differences between IURs are given in Fig.

18: $2 \alpha_{1}\left(2 \alpha_{2}\right) \approx 0$ and $\varphi_{1}-\varphi_{3}=177.2^{\circ} \approx \pi, \varphi_{1}-\varphi_{4}=183.2^{\circ} \approx \pi$ (that means $2 \alpha_{3} \approx \pi$ ).

Corresponding to the experimental displacement data: responses in the $x$ and $y$ directions are equal 


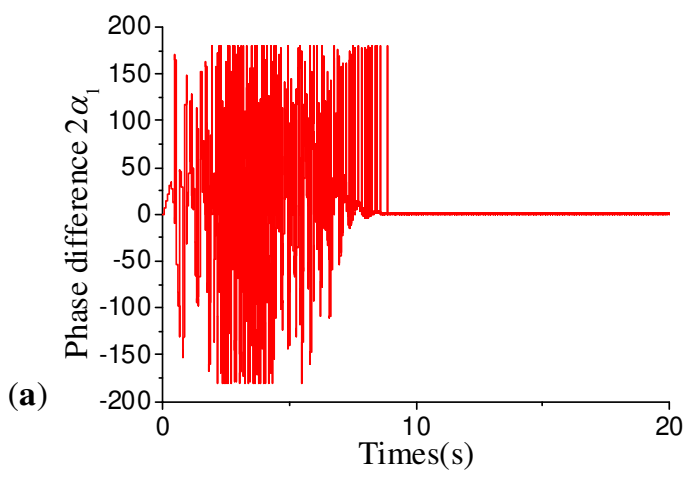

(b)
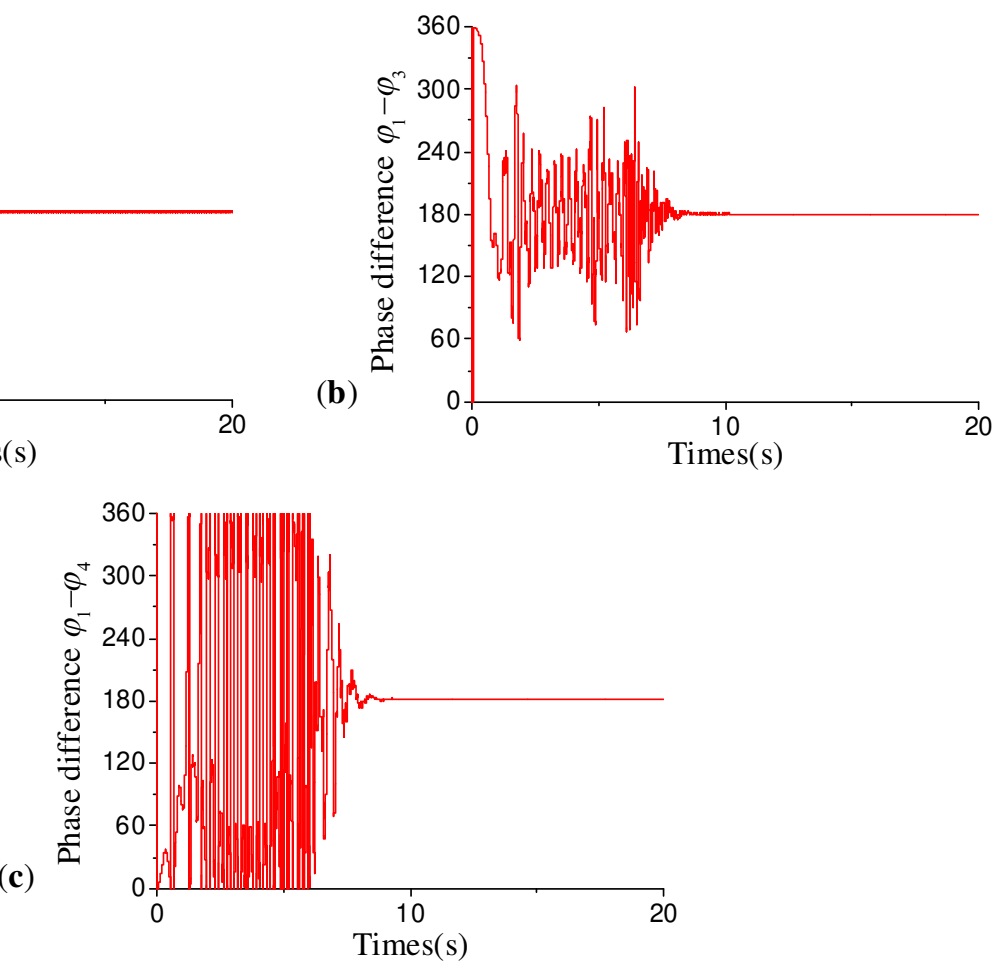

Fig. 18 Experimental phase differences between IURs; (a) Experimental phase difference between exciter 1 and 2; (b) Experimental phase difference between exciter 1 and 3; (c) Experimental phase difference between exciter 1 and 4
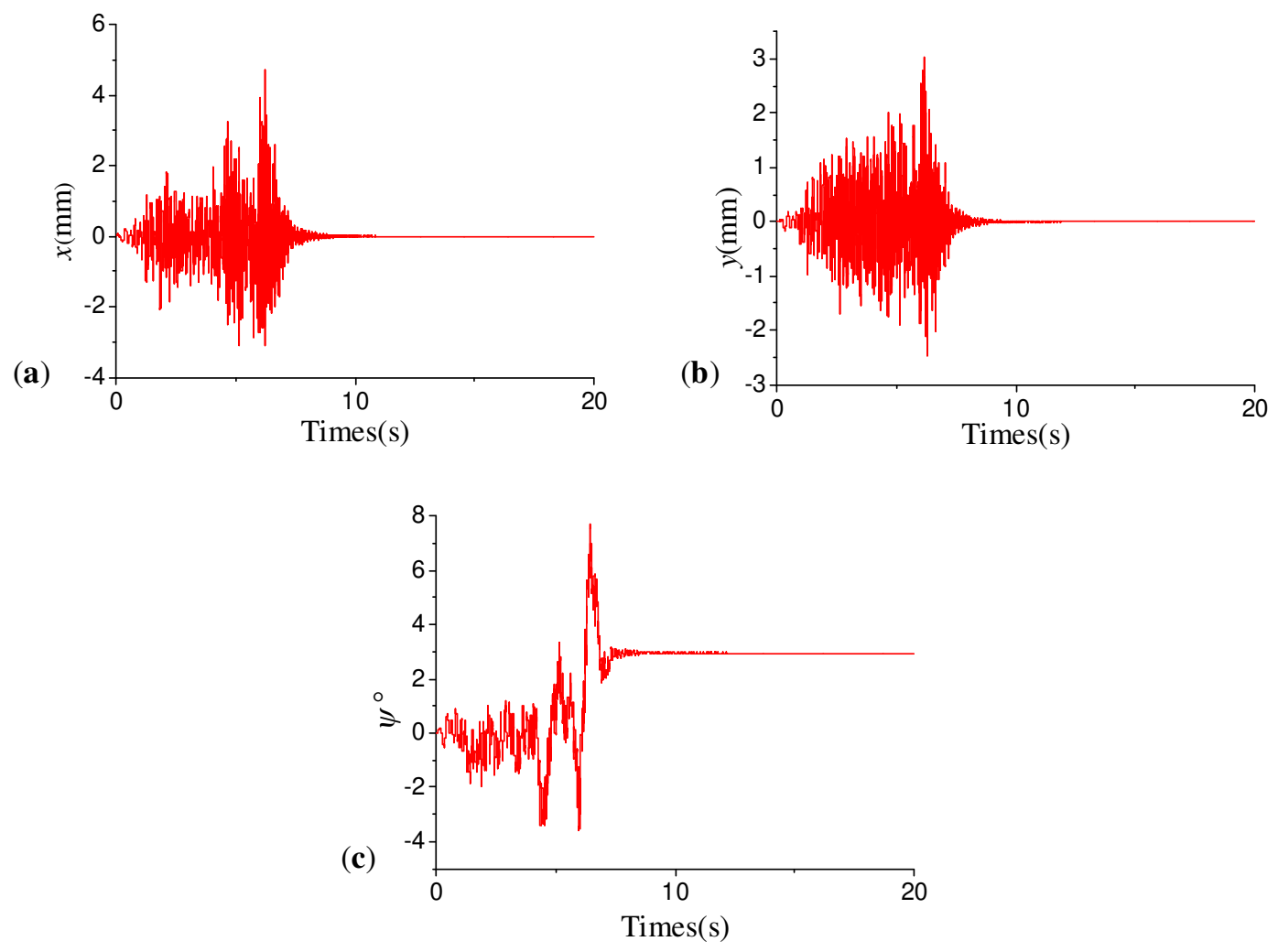

Fig. 19 Experimental displacement results; (a) $x$-direction displacement data; (b) $y$-direction displacement data; (c) $\psi$-direction displacement data 
and gradually approach 0 , and the steady-state response of the $\psi$ direction is at about $2.96^{\circ}$. Through the comparison of the experiment and simulation analysis, we can verify the correctness of the theoretical research.

\section{Conclusions}

In this paper, through a series of theoretical research, we can stress some remarks as follows:

(1) Using the improved small parameter average method, the dimensionless coupling equation of the crusher system is established.

(2) The parameter determination method of eccentric force offsetting self-synchronization under steady state of ultra-resonance is proposed by analyzing the steady-state phase differences, the synchronous capability curve and the stability curve. The parameter interval of the dimensionless parameters is designed: it is verified that the crusher has a set of steady-state phase differences solutions for idle state when $1.414<r_{\mathrm{e}}<2.5$; the synchronous ability coefficient $\zeta_{12}$ increases in this interval and the $\zeta$ value is always greater than 10, indicating that the synchronization performance between motors in this parameter interval is well; the two sets of stability criteria are met simultaneously in the $(1.414,2.5)$ parameter interval.

(3) The design method of working crusher under steady-state of sub-resonance is proposed. It clarifies that the crusher adjusts the system frequency by increasing the materials and changes the phase differences between the four motors to adjust the movement of the body. The movement selection principle of the system is discussed. It is found material equivalent stiffness must not less than 1100 according to the system frequency ratio analysis. Three sets of coefficients are introduced to explore its variation with the material equivalent stiffness and the system damping ratio.

(4) The computer simulation and our experimental verifications fully prove the correctness of the theoretical research and the feasibility of crushing by periodic movement. Based on the 
self-synchronization of dual steady states theoretical model, a new type of crusher can be designed.

Acknowledgements The authors would like to thank National Natural Science Foundation of China

(Grant No. 51775094) for the financial support of the research projects.

Data Availability The authors declare that datasets supporting the conclusions of this study are included within the paper.

\section{Declarations}

Conflict of interest The authors declare that they have no known competing financial interests or personal relationships that could have appeared to influence the work reported in this paper.

\section{Appendix A: The coefficients of equation (16)}

$$
\begin{aligned}
& \chi_{\mathrm{f} 1}=\omega_{m}\left[W_{\mathrm{s} 0}+W_{\mathrm{sc}} \cos 2 \bar{\alpha}_{1}+W_{\mathrm{sc} 13} \cos \left(\bar{\alpha}_{1}-\bar{\alpha}_{2}+2 \bar{\alpha}_{3}\right)+W_{\mathrm{sc} 14} \cos \left(\bar{\alpha}_{1}+\bar{\alpha}_{2}+2 \bar{\alpha}_{3}\right)\right] / 2, \\
& \chi_{\mathrm{a} 1}=\omega_{\mathrm{m}}\left[W_{\mathrm{cc}} \sin 2 \bar{\alpha}_{1}+W_{\mathrm{cc} 13} \sin \left(\bar{\alpha}_{1}-\bar{\alpha}_{2}+2 \bar{\alpha}_{3}\right)+W_{\mathrm{cc} 14} \sin \left(\bar{\alpha}_{1}+\bar{\alpha}_{2}+2 \bar{\alpha}_{3}\right)\right] / 2, \\
& \chi_{\mathrm{f} 2}=\omega_{\mathrm{m}}\left[W_{\mathrm{s} 0}+W_{\mathrm{sc}} \cos 2 \bar{\alpha}_{1}+W_{\mathrm{sc} 23} \cos \left(2 \bar{\alpha}_{3}-\bar{\alpha}_{1}-\bar{\alpha}_{2}\right)+W_{\mathrm{sc} 24} \cos \left(2 \bar{\alpha}_{3}-\bar{\alpha}_{1}+\bar{\alpha}_{2}\right)\right] / 2, \\
& \chi_{\mathrm{a} 2}=\omega_{\mathrm{m}}\left[-W_{\mathrm{cc}} \sin 2 \bar{\alpha}_{1}+W_{\mathrm{cc} 23} \sin \left(2 \bar{\alpha}_{3}-\bar{\alpha}_{1}-\bar{\alpha}_{2}\right)+W_{\mathrm{cc} 24} \sin \left(2 \bar{\alpha}_{3}-\bar{\alpha}_{1}+\bar{\alpha}_{2}\right)\right] / 2, \\
& \chi_{\mathrm{f} 3}=\omega_{\mathrm{m}}\left[W_{\mathrm{s} 0}+W_{\mathrm{sc} 31} \cos \left(\bar{\alpha}_{1}-\bar{\alpha}_{2}+2 \bar{\alpha}_{3}\right)+W_{\mathrm{sc} 32} \cos \left(2 \bar{\alpha}_{3}-\bar{\alpha}_{1}-\bar{\alpha}_{2}\right)+W_{\mathrm{sc}} \cos 2 \bar{\alpha}_{2}\right] / 2, \\
& \chi_{\mathrm{a} 3}=\omega_{\mathrm{m}}\left[W_{\mathrm{cc}} \sin 2 \bar{\alpha}_{2}-W_{\mathrm{cc} 31} \sin \left(\bar{\alpha}_{1}-\bar{\alpha}_{2}+2 \bar{\alpha}_{3}\right)-W_{\mathrm{cc} 32} \sin \left(2 \bar{\alpha}_{3}-\bar{\alpha}_{1}-\bar{\alpha}_{2}\right)\right] / 2, \\
& \chi_{\mathrm{f} 4}=\omega_{\mathrm{m}}\left[W_{\mathrm{s} 0}+W_{\mathrm{sc} 41} \cos \left(\bar{\alpha}_{1}+\bar{\alpha}_{2}+2 \bar{\alpha}_{3}\right)+W_{\mathrm{sc} 42} \cos \left(2 \bar{\alpha}_{3}-\bar{\alpha}_{1}+\bar{\alpha}_{2}\right)+W_{\mathrm{sc}} \cos 2 \bar{\alpha}_{2}\right] / 2, \\
& \chi_{\mathrm{a} 4}=\omega_{\mathrm{m}}\left[-W_{\mathrm{cc} 41} \sin \left(\bar{\alpha}_{1}+\bar{\alpha}_{2}+2 \bar{\alpha}_{3}\right)-W_{\mathrm{cc} 42} \sin \left(2 \bar{\alpha}_{3}-\bar{\alpha}_{1}+\bar{\alpha}_{2}\right)-W_{\mathrm{cc}} \sin 2 \bar{\alpha}_{2}\right] / 2, \\
& \chi_{i i}^{\prime}=W_{\mathrm{c} 0} / 2, \chi_{i i}=\omega_{\mathrm{m}} W_{\mathrm{s} 0}, i=1,2,3,4, \\
& \chi_{12}^{\prime}=\left(W_{\mathrm{cc}} \cos 2 \bar{\alpha}_{1}-W_{\mathrm{sc}} \sin 2 \bar{\alpha}_{1}\right) / 2 \text {, } \\
& \chi_{13}^{\prime}=\left[W_{\mathrm{cc} 13}^{\prime} \cos \left(\bar{\alpha}_{1}-\bar{\alpha}_{2}+2 \bar{\alpha}_{3}\right)-W_{\mathrm{sc} 13}^{\prime} \sin \left(\bar{\alpha}_{1}-\bar{\alpha}_{2}+2 \bar{\alpha}_{3}\right)\right] / 2 \text {, } \\
& \chi_{14}^{\prime}=\left[W_{\mathrm{cc} 14}^{\prime} \cos \left(\bar{\alpha}_{1}+\bar{\alpha}_{2}+2 \bar{\alpha}_{3}\right)-W_{\mathrm{sc} 14}^{\prime} \sin \left(\bar{\alpha}_{1}+\bar{\alpha}_{2}+2 \bar{\alpha}_{3}\right)\right] / 2 \text {, } \\
& \chi_{12}=\omega_{\mathrm{m}}\left(W_{\mathrm{cc}} \sin 2 \bar{\alpha}_{1}+W_{\mathrm{sc}} \cos 2 \bar{\alpha}_{1}\right), \\
& \chi_{13}=\omega_{\mathrm{m}}\left[W_{\mathrm{cc} 13} \sin \left(\bar{\alpha}_{1}-\bar{\alpha}_{2}+2 \bar{\alpha}_{3}\right)+W_{\mathrm{sc} 13} \cos \left(\bar{\alpha}_{1}-\bar{\alpha}_{2}+2 \bar{\alpha}_{3}\right)\right] \text {, } \\
& \chi_{14}=\omega_{\mathrm{m}}\left[W_{\mathrm{cc} 14} \sin \left(\bar{\alpha}_{1}+\bar{\alpha}_{2}+2 \bar{\alpha}_{3}\right)+W_{\mathrm{sc} 14} \cos \left(\bar{\alpha}_{1}+\bar{\alpha}_{2}+2 \bar{\alpha}_{3}\right)\right] \text {, } \\
& \chi_{21}^{\prime}=\left(W_{\mathrm{cc}} \cos 2 \bar{\alpha}_{1}+W_{\mathrm{sc}} \sin 2 \bar{\alpha}_{1}\right) / 2, \\
& \chi_{23}^{\prime}=\left[W_{\mathrm{cc} 23}^{\prime} \cos \left(2 \bar{\alpha}_{3}-\bar{\alpha}_{1}-\bar{\alpha}_{2}\right)-W_{\mathrm{sc} 23}^{\prime} \sin \left(2 \bar{\alpha}_{3}-\bar{\alpha}_{1}-\bar{\alpha}_{2}\right)\right] / 2 \text {, } \\
& \chi_{24}^{\prime}=\left[W_{\mathrm{c} 0} \cos \left(2 \bar{\alpha}_{3}-\bar{\alpha}_{1}+\bar{\alpha}_{2}\right)-W_{\mathrm{sc}} \sin \left(2 \bar{\alpha}_{3}-\bar{\alpha}_{1}+\bar{\alpha}_{2}\right)\right] / 2, \\
& \chi_{21}=\omega_{\mathrm{m}}\left(W_{\mathrm{sc}} \cos 2 \bar{\alpha}_{1}-W_{\mathrm{cc}} \sin 2 \bar{\alpha}_{1}\right), \\
& \chi_{23}=\omega_{\mathrm{m}}\left[W_{\mathrm{sc} 23} \cos \left(2 \bar{\alpha}_{3}-\bar{\alpha}_{1}-\bar{\alpha}_{2}\right)+W_{\mathrm{cc} 23} \sin \left(2 \bar{\alpha}_{3}-\bar{\alpha}_{1}-\bar{\alpha}_{2}\right)\right] \text {, }
\end{aligned}
$$




$$
\begin{aligned}
& \chi_{24}=\omega_{\mathrm{m}}\left[W_{\mathrm{sc} 24} \cos \left(2 \bar{\alpha}_{3}-\bar{\alpha}_{1}+\bar{\alpha}_{2}\right)+W_{\mathrm{cc} 24} \sin \left(2 \bar{\alpha}_{3}-\bar{\alpha}_{1}+\bar{\alpha}_{2}\right)\right] \text {, } \\
& \chi_{31}^{\prime}=\left[W_{\mathrm{c} 0} \cos \left(\bar{\alpha}_{1}-\bar{\alpha}_{2}+2 \bar{\alpha}_{3}\right)+W_{\mathrm{s} 0} \sin \left(\bar{\alpha}_{1}-\bar{\alpha}_{2}+2 \bar{\alpha}_{3}\right)\right] / 2, \\
& \chi_{32}^{\prime}=\left[W_{\mathrm{cc} 32}^{\prime} \cos \left(2 \bar{\alpha}_{3}-\bar{\alpha}_{1}-\bar{\alpha}_{2}\right)+W_{\mathrm{sc} 32}^{\prime} \sin \left(2 \bar{\alpha}_{3}-\bar{\alpha}_{1}-\bar{\alpha}_{2}\right)\right] / 2, \\
& \chi_{34}^{\prime}=\left(W_{\mathrm{cc}} \cos 2 \bar{\alpha}_{2}-W_{\mathrm{sc}} \sin 2 \bar{\alpha}_{2}\right) / 2, \\
& \chi_{31}=\omega_{\mathrm{m}}\left[W_{\mathrm{sc} 31} \cos \left(\bar{\alpha}_{1}-\bar{\alpha}_{2}+2 \bar{\alpha}_{3}\right)-W_{\mathrm{cc} 31} \sin \left(\bar{\alpha}_{1}-\bar{\alpha}_{2}+2 \bar{\alpha}_{3}\right)\right] \text {, } \\
& \chi_{32}=\omega_{\mathrm{m}}\left[W_{\mathrm{sc} 32} \cos \left(2 \bar{\alpha}_{3}-\bar{\alpha}_{1}-\bar{\alpha}_{2}\right)-W_{\mathrm{cc} 32} \sin \left(2 \bar{\alpha}_{3}-\bar{\alpha}_{1}-\bar{\alpha}_{2}\right)\right] \text {, } \\
& \chi_{34}=\omega_{\mathrm{m}}\left(W_{\mathrm{sc}} \cos 2 \bar{\alpha}_{2}+W_{\mathrm{cc}} \sin 2 \bar{\alpha}_{2}\right), \\
& \chi_{41}^{\prime}=\left[W_{\mathrm{cc} 41}^{\prime} \cos \left(\bar{\alpha}_{1}+\bar{\alpha}_{2}+2 \bar{\alpha}_{3}\right)+W_{\mathrm{sc} 41}^{\prime} \sin \left(\bar{\alpha}_{1}+\bar{\alpha}_{2}+2 \bar{\alpha}_{3}\right)\right] / 2, \\
& \chi_{42}^{\prime}=\left[W_{\mathrm{cc} 42}^{\prime} \cos \left(2 \bar{\alpha}_{3}-\bar{\alpha}_{1}+\bar{\alpha}_{2}\right)+W_{\mathrm{sc} 42}^{\prime} \sin \left(2 \bar{\alpha}_{3}-\bar{\alpha}_{1}+\bar{\alpha}_{2}\right)\right] / 2, \\
& \chi_{43}^{\prime}=\left(W_{\mathrm{cc}} \cos 2 \bar{\alpha}_{2}+W_{\mathrm{sc}} \sin 2 \bar{\alpha}_{2}\right) / 2, \\
& \chi_{41}=\omega_{\mathrm{m}}\left[W_{\mathrm{sc} 41} \cos \left(\bar{\alpha}_{1}+\bar{\alpha}_{2}+2 \bar{\alpha}_{3}\right)-W_{\mathrm{cc} 41} \sin \left(\bar{\alpha}_{1}+\bar{\alpha}_{2}+2 \bar{\alpha}_{3}\right)\right] \text {, } \\
& \chi_{42}=\omega_{\mathrm{m}}\left[W_{\mathrm{sc} 42} \cos \left(2 \bar{\alpha}_{3}-\bar{\alpha}_{1}+\bar{\alpha}_{2}\right)-W_{\mathrm{cc} 42} \sin \left(2 \bar{\alpha}_{3}-\bar{\alpha}_{1}+\bar{\alpha}_{2}\right)\right] \text {, } \\
& \chi_{43}=\omega_{\mathrm{m}}\left(W_{\mathrm{sc}} \cos 2 \bar{\alpha}_{2}-W_{\mathrm{cc}} \sin 2 \bar{\alpha}_{2}\right), \\
& W_{\mathrm{c} 0}=r_{\mathrm{m}}\left(\mu_{x} \cos \gamma_{x}+\mu_{y} \cos \gamma_{y}+r_{\mathrm{e}}^{2} \mu_{\psi} \cos \gamma_{\psi}\right) \text {, } \\
& W_{\mathrm{cc}}=r_{\mathrm{m}}\left(\mu_{x} \cos \gamma_{x}+\mu_{y} \cos \gamma_{y}-r_{\mathrm{e}}^{2} \mu_{\psi} \cos \gamma_{\psi}\right) \text {, } \\
& W_{\mathrm{s} 0}=r_{\mathrm{m}}\left(\mu_{x} \sin \gamma_{x}+\mu_{y} \sin \gamma_{y}+r_{\mathrm{e}}^{2} \mu_{\psi} \sin \gamma_{\psi}\right) \text {, } \\
& W_{\mathrm{sc}}=r_{\mathrm{m}}\left(\mu_{x} \sin \gamma_{x}+\mu_{y} \sin \gamma_{y}-r_{\mathrm{e}}^{2} \mu_{\psi} \sin \gamma_{\psi}\right) \text {. } \\
& W_{\mathrm{cc} 13}=W_{\mathrm{cc} 31}=r_{\mathrm{m}}\left[\mu_{x} \cos \gamma_{x}+\mu_{y} \cos \gamma_{y}+r_{\mathrm{e}}^{2} \mu_{\psi} \cos \gamma_{\psi} \cot \left(\bar{\alpha}_{1}-\bar{\alpha}_{2}+2 \bar{\alpha}_{3}\right)\right] \text {, } \\
& W_{\mathrm{sc} 13}=W_{\mathrm{sc} 31}=r_{\mathrm{m}}\left[\mu_{x} \sin \gamma_{x}+\mu_{y} \sin \gamma_{y}-r_{\mathrm{e}}^{2} \mu_{\psi} \sin \gamma_{\psi} \tan \left(\bar{\alpha}_{1}-\bar{\alpha}_{2}+2 \bar{\alpha}_{3}\right)\right] \text {, } \\
& W_{\mathrm{cc} 14}=W_{\mathrm{cc} 41}=r_{\mathrm{m}}\left[\mu_{x} \cos \gamma_{x}+\mu_{y} \cos \gamma_{y}-r_{\mathrm{e}}^{2} \mu_{\psi} \cos \gamma_{\psi} \cot \left(\bar{\alpha}_{1}+\bar{\alpha}_{2}+2 \bar{\alpha}_{3}\right)\right] \text {, } \\
& W_{\mathrm{sc} 14}=W_{\mathrm{sc} 41}=r_{\mathrm{m}}\left[\mu_{x} \sin \gamma_{x}+\mu_{y} \sin \gamma_{y}+r_{\mathrm{e}}^{2} \mu_{\psi} \sin \gamma_{\psi} \tan \left(\bar{\alpha}_{1}+\bar{\alpha}_{2}+2 \bar{\alpha}_{3}\right)\right] \text {, } \\
& W_{\mathrm{cc} 23}=W_{\mathrm{cc} 32}=r_{\mathrm{m}}\left[\mu_{x} \cos \gamma_{x}+\mu_{y} \cos \gamma_{y}-r_{\mathrm{e}}^{2} \mu_{\psi} \cos \gamma_{\psi} \cot \left(2 \bar{\alpha}_{3}-\bar{\alpha}_{1}-\bar{\alpha}_{2}\right)\right] \text {, } \\
& W_{\mathrm{sc} 23}=W_{\mathrm{sc} 32}=r_{\mathrm{m}}\left[\mu_{x} \sin \gamma_{x}+\mu_{y} \sin \gamma_{y}+r_{\mathrm{e}}^{2} \mu_{\psi} \sin \gamma_{\psi} \tan \left(2 \bar{\alpha}_{3}-\bar{\alpha}_{1}-\bar{\alpha}_{2}\right)\right] \text {, } \\
& W_{\mathrm{cc} 24}=W_{\mathrm{cc} 42}=r_{\mathrm{m}}\left[\mu_{x} \cos \gamma_{x}+\mu_{y} \cos \gamma_{y}+r_{\mathrm{e}}^{2} \mu_{\psi} \cos \gamma_{\psi} \cot \left(2 \bar{\alpha}_{3}-\bar{\alpha}_{1}+\bar{\alpha}_{2}\right)\right] \text {, } \\
& W_{\mathrm{sc} 24}=W_{\mathrm{sc} 42}=r_{\mathrm{m}}\left[\mu_{x} \sin \gamma_{x}+\mu_{y} \sin \gamma_{y}-r_{\mathrm{e}}^{2} \mu_{\psi} \sin \gamma_{\psi} \tan \left(2 \bar{\alpha}_{3}-\bar{\alpha}_{1}+\bar{\alpha}_{2}\right)\right] \text {, } \\
& W_{\mathrm{cc} 13}^{\prime}=W_{\mathrm{cc} 31}^{\prime}=r_{\mathrm{m}}\left[\mu_{x} \cos \gamma_{x}+\mu_{y} \cos \gamma_{y}-r_{\mathrm{e}}^{2} \mu_{\psi} \cos \gamma_{\psi} \tan \left(\bar{\alpha}_{1}-\bar{\alpha}_{2}+2 \bar{\alpha}_{3}\right)\right] \text {, } \\
& W_{\mathrm{sc} 13}^{\prime}=W_{\mathrm{sc} 31}^{\prime}=r_{\mathrm{m}}\left[\mu_{x} \sin \gamma_{x}+\mu_{y} \sin \gamma_{y}+r_{\mathrm{e}}^{2} \mu_{\psi} \sin \gamma_{\psi} \cot \left(\bar{\alpha}_{1}-\bar{\alpha}_{2}+2 \bar{\alpha}_{3}\right)\right] \text {, } \\
& W_{\mathrm{cc} 14}^{\prime}=W_{\mathrm{cc} 41}^{\prime}=r_{\mathrm{m}}\left[\mu_{x} \cos \gamma_{x}+\mu_{y} \cos \gamma_{y}+r_{\mathrm{e}}^{2} \mu_{\psi} \cos \gamma_{\psi} \tan \left(\bar{\alpha}_{1}+\bar{\alpha}_{2}+2 \bar{\alpha}_{3}\right)\right] \text {, } \\
& W_{\mathrm{sc} 14}^{\prime}=W_{\mathrm{sc} 41}^{\prime}=r_{\mathrm{m}}\left[\mu_{x} \sin \gamma_{x}+\mu_{y} \sin \gamma_{y}-r_{\mathrm{e}}^{2} \mu_{\psi} \sin \gamma_{\psi} \cot \left(\bar{\alpha}_{1}+\bar{\alpha}_{2}+2 \bar{\alpha}_{3}\right)\right] \text {, } \\
& W_{\mathrm{cc} 23}^{\prime}=W_{\mathrm{cc} 32}^{\prime}=r_{\mathrm{m}}\left[\mu_{x} \cos \gamma_{x}+\mu_{y} \cos \gamma_{y}+r_{\mathrm{e}}^{2} \mu_{\psi} \cos \gamma_{\psi} \tan \left(2 \bar{\alpha}_{3}-\bar{\alpha}_{1}-\bar{\alpha}_{2}\right)\right] \text {, }
\end{aligned}
$$




$$
\begin{aligned}
& W_{\mathrm{sc} 23}^{\prime}=W_{\mathrm{sc} 32}^{\prime}=r_{\mathrm{m}}\left[\mu_{x} \sin \gamma_{x}+\mu_{y} \sin \gamma_{y}-r_{\mathrm{e}}^{2} \mu_{\psi} \sin \gamma_{\psi} \cot \left(2 \bar{\alpha}_{3}-\bar{\alpha}_{1}-\bar{\alpha}_{2}\right)\right], \\
& W_{\mathrm{cc} 24}^{\prime}=W_{\mathrm{cc} 42}^{\prime}=r_{\mathrm{m}}\left[\mu_{x} \cos \gamma_{x}+\mu_{y} \cos \gamma_{y}-r_{\mathrm{e}}^{2} \mu_{\psi} \cos \gamma_{\psi} \tan \left(2 \bar{\alpha}_{3}-\bar{\alpha}_{1}+\bar{\alpha}_{2}\right)\right], \\
& W_{\mathrm{sc} 24}^{\prime}=W_{\mathrm{sc} 42}^{\prime}=r_{\mathrm{m}}\left[\mu_{x} \sin \gamma_{x}+\mu_{y} \sin \gamma_{y}+r_{\mathrm{e}}^{2} \mu_{\psi} \sin \gamma_{\psi} \cot \left(2 \bar{\alpha}_{3}-\bar{\alpha}_{1}+\bar{\alpha}_{2}\right)\right] .
\end{aligned}
$$

\section{Appendix B: Elements of the matrices $E$ and $D$}

$$
\begin{aligned}
& e_{11}=k_{\mathrm{e} 01}\left(1+\rho_{1}\right)+k_{\mathrm{e} 02}\left(1-\rho_{1}\right), \\
& e_{12}=\left(k_{\mathrm{e} 01}-k_{\mathrm{e} 02}\right) \rho_{2}, \\
& e_{13}=\left(k_{\mathrm{e} 01}-k_{\mathrm{e} 02}\right)\left(1+\rho_{3}\right) \text {, } \\
& e_{21}=\left(k_{\mathrm{e} 03}-k_{\mathrm{e} 04}\right) \rho_{1}, \\
& e_{22}=k_{\mathrm{e} 03}\left(1+\rho_{2}\right)+k_{\mathrm{e} 04}\left(1-\rho_{2}\right) \text {, } \\
& e_{23}=\left(k_{\mathrm{e} 03}-k_{\mathrm{e} 04}\right)\left(\rho_{3}-1\right), \\
& e_{31}=k_{\mathrm{e} 01}\left(1+\rho_{1}\right)+k_{\mathrm{e} 02}\left(\rho_{1}-1\right)-\left(k_{\mathrm{e} 03}+k_{\mathrm{e} 04}\right) \rho_{1} \text {, } \\
& e_{32}=\left(k_{\mathrm{e} 01}+k_{\mathrm{e} 02}-k_{\mathrm{e} 03}-k_{\mathrm{e} 04}\right) \rho_{2}+k_{\mathrm{e} 04}-k_{\mathrm{e} 03}, \\
& e_{33}=\left(k_{\mathrm{e} 01}+k_{\mathrm{e} 02}-k_{\mathrm{e} 03}-k_{\mathrm{e} 04}\right) \rho_{3}+k_{\mathrm{e} 01}+k_{\mathrm{e} 02}+k_{\mathrm{e} 03}+k_{\mathrm{e} 04} \text {, } \\
& d_{11}=\left(-\omega_{\mathrm{m} 0}^{2} / 2\right)\left[4 W_{\mathrm{cc}} \cos 2 \bar{\alpha}_{10}+W_{\mathrm{cc} 23} \cos \left(2 \bar{\alpha}_{30}-\bar{\alpha}_{10}-\bar{\alpha}_{20}\right)+r_{\mathrm{m}} r_{\mathrm{e}}^{2} \mu_{\psi} \csc \left(2 \bar{\alpha}_{30}-\bar{\alpha}_{10}-\bar{\alpha}_{20}\right)\right. \\
& +W_{\mathrm{cc} 24} \cos \left(2 \bar{\alpha}_{30}-\bar{\alpha}_{10}+\bar{\alpha}_{20}\right)-r_{\mathrm{m}} r_{\mathrm{e}}^{2} \mu_{\psi} \csc \left(2 \bar{\alpha}_{30}-\bar{\alpha}_{10}+\bar{\alpha}_{20}\right) \\
& +W_{\mathrm{cc} 13} \cos \left(\bar{\alpha}_{10}-\bar{\alpha}_{20}+2 \bar{\alpha}_{30}\right)-r_{\mathrm{m}} r_{\mathrm{e}}^{2} \mu_{\psi} \csc \left(\bar{\alpha}_{10}-\bar{\alpha}_{20}+2 \bar{\alpha}_{30}\right) \\
& \left.+W_{\mathrm{cc} 14} \cos \left(\bar{\alpha}_{10}+\bar{\alpha}_{20}+2 \bar{\alpha}_{30}\right)+r_{\mathrm{m}} r_{\mathrm{e}}^{2} \mu_{\psi} \csc \left(\bar{\alpha}_{10}+\bar{\alpha}_{20}+2 \bar{\alpha}_{30}\right)\right] \text {, } \\
& d_{12}=d_{21}=\left(-\omega_{\mathrm{m} 0}^{2} / 2\right)\left[W_{\mathrm{cc} 23} \cos \left(2 \bar{\alpha}_{30}-\bar{\alpha}_{10}-\bar{\alpha}_{20}\right)+r_{\mathrm{m}} r_{\mathrm{e}}^{2} \mu_{\psi} \csc \left(2 \bar{\alpha}_{30}-\bar{\alpha}_{10}-\bar{\alpha}_{20}\right)\right. \\
& -W_{\mathrm{cc} 24} \cos \left(2 \bar{\alpha}_{30}-\bar{\alpha}_{10}+\bar{\alpha}_{20}\right)+r_{\mathrm{m}} r_{\mathrm{e}}^{2} \mu_{\psi} \csc \left(2 \bar{\alpha}_{30}-\bar{\alpha}_{10}+\bar{\alpha}_{20}\right) \\
& -W_{\mathrm{cc} 13} \cos \left(\bar{\alpha}_{10}-\bar{\alpha}_{20}+2 \bar{\alpha}_{30}\right)+r_{\mathrm{m}} r_{\mathrm{e}}^{2} \mu_{\psi} \csc \left(\bar{\alpha}_{10}-\bar{\alpha}_{20}+2 \bar{\alpha}_{30}\right) \\
& \left.+W_{\mathrm{cc} 14} \cos \left(\bar{\alpha}_{10}+\bar{\alpha}_{20}+2 \bar{\alpha}_{30}\right)+r_{\mathrm{m}} r_{\mathrm{e}}^{2} \mu_{\psi} \csc \left(\bar{\alpha}_{10}+\bar{\alpha}_{20}+2 \bar{\alpha}_{30}\right)\right], \\
& d_{13}=d_{31}=-\omega_{\mathrm{m} 0}^{2}\left[-W_{\mathrm{cc} 23} \cos \left(2 \bar{\alpha}_{30}-\bar{\alpha}_{10}-\bar{\alpha}_{20}\right)-r_{\mathrm{m}} r_{\mathrm{e}}^{2} \mu_{\psi} \csc \left(2 \bar{\alpha}_{30}-\bar{\alpha}_{10}-\bar{\alpha}_{20}\right)\right. \\
& -W_{\mathrm{cc} 24} \cos \left(2 \bar{\alpha}_{30}-\bar{\alpha}_{10}+\bar{\alpha}_{20}\right)+r_{\mathrm{m}} r_{\mathrm{e}}^{2} \mu_{\psi} \csc \left(2 \bar{\alpha}_{30}-\bar{\alpha}_{10}+\bar{\alpha}_{20}\right) \\
& +W_{\mathrm{cc} 13} \cos \left(\bar{\alpha}_{10}-\bar{\alpha}_{20}+2 \bar{\alpha}_{30}\right)-r_{\mathrm{m}} r_{\mathrm{e}}^{2} \mu_{\psi} \csc \left(\bar{\alpha}_{10}-\bar{\alpha}_{20}+2 \bar{\alpha}_{30}\right) \\
& \left.+W_{\mathrm{cc} 14} \cos \left(\bar{\alpha}_{10}+\bar{\alpha}_{20}+2 \bar{\alpha}_{30}\right)+r_{\mathrm{m}} r_{\mathrm{e}}^{2} \mu_{\psi} \csc \left(\bar{\alpha}_{10}+\bar{\alpha}_{20}+2 \bar{\alpha}_{30}\right)\right] \\
& d_{22}=\left(-\omega_{\mathrm{m} 0}^{2} / 2\right)\left[4 W_{\mathrm{cc}} \cos 2 \bar{\alpha}_{20}+W_{\mathrm{cc} 14} \cos \left(\bar{\alpha}_{10}+\bar{\alpha}_{20}+2 \bar{\alpha}_{30}\right)+r_{\mathrm{m}} r_{\mathrm{e}}^{2} \mu_{\psi} \csc \left(\bar{\alpha}_{10}+\bar{\alpha}_{20}+2 \bar{\alpha}_{30}\right)\right. \\
& +W_{\mathrm{cc} 24} \cos \left(2 \bar{\alpha}_{30}-\bar{\alpha}_{10}+\bar{\alpha}_{20}\right)-r_{\mathrm{m}} r_{\mathrm{e}}^{2} \mu_{\psi} \csc \left(2 \bar{\alpha}_{30}-\bar{\alpha}_{10}+\bar{\alpha}_{20}\right) \\
& +W_{\mathrm{cc} 13} \cos \left(\bar{\alpha}_{10}-\bar{\alpha}_{20}+2 \bar{\alpha}_{30}\right)-r_{\mathrm{m}} r_{\mathrm{e}}^{2} \mu_{\psi} \csc \left(\bar{\alpha}_{10}-\bar{\alpha}_{20}+2 \bar{\alpha}_{30}\right) \\
& \left.+W_{\mathrm{cc} 23} \cos \left(2 \bar{\alpha}_{30}-\bar{\alpha}_{10}-\bar{\alpha}_{20}\right)+r_{\mathrm{m}} r_{\mathrm{e}}^{2} \mu_{\psi} \csc \left(2 \bar{\alpha}_{30}-\bar{\alpha}_{10}-\bar{\alpha}_{20}\right)\right],
\end{aligned}
$$




$$
\begin{aligned}
d_{23}=d_{32} & =-\omega_{\mathrm{m} 0}^{2}\left[W_{\mathrm{cc} 14} \cos \left(\bar{\alpha}_{10}+\bar{\alpha}_{20}+2 \bar{\alpha}_{30}\right)+r_{\mathrm{m}} r_{\mathrm{e}}^{2} \mu_{\psi} \csc \left(\bar{\alpha}_{10}+\bar{\alpha}_{20}+2 \bar{\alpha}_{30}\right)\right. \\
& +W_{\mathrm{cc} 24} \cos \left(2 \bar{\alpha}_{30}-\bar{\alpha}_{10}+\bar{\alpha}_{20}\right)-r_{\mathrm{m}} r_{\mathrm{e}}^{2} \mu_{\psi} \csc \left(2 \bar{\alpha}_{30}-\bar{\alpha}_{10}+\bar{\alpha}_{20}\right) \\
& -W_{\mathrm{cc} 13} \cos \left(\bar{\alpha}_{10}-\bar{\alpha}_{20}+2 \bar{\alpha}_{30}\right)+r_{\mathrm{m}} r_{\mathrm{e}}^{2} \mu_{\psi} \csc \left(\bar{\alpha}_{10}-\bar{\alpha}_{20}+2 \bar{\alpha}_{30}\right) \\
& \left.-W_{\mathrm{cc} 23} \cos \left(2 \bar{\alpha}_{30}-\bar{\alpha}_{10}-\bar{\alpha}_{20}\right)-r_{\mathrm{m}} r_{\mathrm{e}}^{2} \mu_{\psi} \csc \left(2 \bar{\alpha}_{30}-\bar{\alpha}_{10}-\bar{\alpha}_{20}\right)\right] \\
d_{33}= & -2 \omega_{\mathrm{m} 0}^{2}\left[W_{\mathrm{cc} 13} \cos \left(\bar{\alpha}_{10}-\bar{\alpha}_{20}+2 \bar{\alpha}_{30}\right)-r_{\mathrm{m}} r_{\mathrm{e}}^{2} \mu_{\psi} \csc \left(\bar{\alpha}_{10}-\bar{\alpha}_{20}+2 \bar{\alpha}_{30}\right)\right. \\
& +W_{\mathrm{cc} 23} \cos \left(2 \bar{\alpha}_{30}-\bar{\alpha}_{10}-\bar{\alpha}_{20}\right)+r_{\mathrm{m}} r_{\mathrm{e}}^{2} \mu_{\psi} \csc \left(2 \bar{\alpha}_{30}-\bar{\alpha}_{10}-\bar{\alpha}_{20}\right) \\
& +W_{\mathrm{cc} 14} \cos \left(\bar{\alpha}_{10}+\bar{\alpha}_{20}+2 \bar{\alpha}_{30}\right)+r_{\mathrm{m}} r_{\mathrm{e}}^{2} \mu_{\psi} \csc \left(\bar{\alpha}_{10}+\bar{\alpha}_{20}+2 \bar{\alpha}_{30}\right) \\
& \left.+W_{\mathrm{cc} 24} \cos \left(2 \bar{\alpha}_{30}-\bar{\alpha}_{10}+\bar{\alpha}_{20}\right)-r_{\mathrm{m}} r_{\mathrm{e}}^{2} \mu_{\psi} \csc \left(2 \bar{\alpha}_{30}-\bar{\alpha}_{10}+\bar{\alpha}_{20}\right)\right] .
\end{aligned}
$$

\section{References}

[1] I.I. Blekhman, Synchronization in Science and Technology, ASME Press, New York. 1988.

[2] I.I. Blekhman, Synchronization of Dynamical Systems, Nauka, Moscow. 1971. (in Russian)

[3] I.I. Blekhman, N.P. Yaroshevich, Extension of the domain of applicability of the integral stability criterion (extremum property) in synchronization problems, Journal of Applied Mathematics and Mechanics. 68 (2004) 839-846. (balthazar05)

[4] I.I. Blekhman, Selected Topics in Vibrational Mechanics. Singapore: World Scientific. 2004.

[5] I.I. Blekhman, V.S.Sorokin, On the separation of fast and slow motions in mechanical systems with high-frequency modulation of the dissipation coefficient, Journal of Sound and Vibration. 329 (2010) 4936-4949. (blekhman02)

[6] B.C. Wen, H. Zhang, S.Y. Liu et al, Theory and Techniques of Vibrating Machinery and their Applications, Beijing: Science Press. 2010.

[7] B.C. Wen, J. Fan, C.Y. Zhao et al, Vibratory Synchronization and Controlled Synchronization in Engineering, Science Press, Beijing. 2009.

[8] Wen B C, Recent development of vibration utilization engineering, Frontiers Mech Eng China. $3(2008) 1-9$.

[9] C.Y. Zhao, D.G. Wang, H. Zhang et al, Capture frequency of vibrating system with two-motor 
drives rotating in the same direction, Chinese Journal of Applied Mechanics. 26 (2009) 283-287. (in Chinese)

[10]C.Y. Zhao, B.C. Wen, X.L. Zhang, Synchronization of the four identical unbalanced rotors in a vibrating system of plane motion, Science China E: Technological Sciences. 53 (2010) $405-422$.

[11]C.Y. Zhao, Q.H. Zhao, Y.M. Zhang et al, Synchronization of two non-identical coupled exciters in a non-resonant vibrating system of plane motion, Journal of Mechanical Science and Technology. 25 (2011) 1253-1260.

[12]C.Y. Zhao, H.T. Zhu, T.J. Bai et al, Synchronization of two non-identical coupled exciters in a non-resonant vibrating system of linear motion. Part I: Theoretical analysis, Shock and Vibration. 16 (2009) 505-515.

[13]C.Y. Zhao, H.T. Zhu, Y.M. Zhang et al, Synchronization of two coupled exciters in a vibrating system of spatial motion, Acta Mech Sin. 26 (2010) 477-493.

[14]C.Y. Zhao, Y.M. Zhang, B.C. Wen, Synchronization and general dynamic symmetry of a vibrating system with two exciters rotating in opposite directions, Chinese Physics B. 53 (2010) $405-422$.

[15]D.W. Gu, J.Q. Zhang, B.C. Wen et al, Theoretical and experimental investigation on controlled synchronization of four co-rotating coupled exciters driven by induction motors in a vibrating system, Proc IMechE Part C:J Mechanical Engineering Science. 233 (2019) 4556-4576.

[16]X.X. Kong, C. Zhou, B.C. Wen, Composite synchronization of four exciters driven by induction motors in a vibration system, Meccanica. 55 (2020) 2107-2133.

[17]H. Peng, Y.J. Hou, P. Fang, M. Zou, Z.L. Zhang, Synchronization analysis of the anti-resonance system with three exciters, Applied Mathematical Modelling. 97 (2021) 96-112. 
[18] Y.J. Hou, H. Peng, P. Fang and M. Zou, Synchronous characteristic of three homodromy motors in vibrating isolation system, Journal of Mechanical Science and Technology. 35-1 (2021) 45-60.

[19]X.L. Zhang, B.C. Wen, C.Y. Zhao, Theoretical, Numerical and Experimental Study on Synchronization of Three Identical Exciters in a Vibrating System, Chinese Journal of Mechanical Engineering. 26-4 (2013) 746-757.

[20]X.L. Zhang, B.C. Wen, C.Y. Zhao, Experimental investigation on synchronization of three co-rotating non-identical coupled exciters driven by three motors, Journal of Sound and Vibration. 333 (2014) 2898-2908.

[21]A.E. Shokhin, A.N. Nikiforov, On the Rational Dynamic Modes of Vibrating Machineswith an Unbalanced Vibration Exciter of Limited Power, Journal of Machinery Manufacture and Reliability. 46-5 (2017) 426-433.

[22]I.I. Blekhman, V.B. Vasilkov, N.P. Yaroshevich, On Some Opportunities for Improving Vibration Machines with Self-Synchronizing Inert Vibration Exciters, Journal of Machinery Manufacture and Reliability. 42-3 (2013) 192-195.

[23]A.E. Shokhin, K.V. Krestnikovskii, A.N. Nikiforov, On self-synchronization of inertial vibration exciters in a vibroimpact three-mass system, IOP Conf. Series: Materials Science and Engineering. 1129 (2021) 012041.

[24]A.E. Shokhin, Self-Synchronization of a Vibrating Jaw Crusher with Allowance for Interaction with the Medium Processed, Journal of Machinery Manufacture and Reliability. 49-6 (2020) $500-510$.

[25]X.H. Zhang, Q.L. Zhang, Control Theory of Nonlinear Differential Algebraic System and Its Applications, Beijing: Science Press. 2007. (in Chinese) 